\title{
Hearing Attacks in Network Data: an Effectiveness Study
}

\author{
Louise Axon, Jassim Happa, Michael Goldsmith, and Sadie Creese \\ Department of Computer Science, University of Oxford, Parks Road, Oxford, OX1 3PR \\ (email: \{firstname.lastname\}@cs.ox.ac.uk)
}

\begin{abstract}
Sonification, in which data is represented as sound, can be used to turn network attacks and network-security information into audio signals. This could complement the range of security-monitoring tools currently used in Security Operations Centres (SOCs). Prior work in sonification for network monitoring has not assessed the effectiveness of the technique for enabling users to monitor network-security information. To this end, we aim to investigate the viability of using sonified network datasets to enable humans to detect (recognise the presence of some) and identify (understand the type of) network attacks. In this paper we report the results of a user study in which we assessed the utility of a network-traffic sonification system for representing network attacks. Our results show that by listening to the sonified network data, participants could detect attacks accurately and efficiently, including combinations of attacks, and identify the types of attacks. Musical experience had no significant effect on the ability of participants to use the sonification, and participants could detect attacks without training, yet improved performance through training. The results support the potential of sonification for use in network-security monitoring tasks.
\end{abstract}

Keywords: Sonification, Network-security monitoring, Intrusion detection, Network attacks, Security operations centres

\section{Introduction}

Organisations are frequently targeted by network attacks, which vary widely in motivation, characteristics and scale. According to IBM, client organisations experienced on average 1,019 attacks in 2016 [1]. The timely and accurate detection of threats is crucial to maintaining confidentiality, integrity and availability of network data and functionality. In large organisations, this is the function of Security Operations Centres (SOCs), in which security analysts work, aided by security-monitoring tools, to detect and prevent cyber attacks. The threats faced are evolving in nature constantly and detection is often very challenging; as such there is a constant need for improved monitoring tools and methods in SOCs. 
Automated approaches to attack detection are widely used in SOCs to help analysts deal with the volume of attacks. These include Intrusion Detection Systems (IDSs), network firewalls, and statistical or Machine Learning-based approaches to anomaly detection [2]. While vital to operations, these automated approaches can be inaccurate [3], and automated approaches alone cannot be relied on to maintain organisational cybersecurity. Human security practitioners play an important role in determining inaccuracies in alerting from such tools, identifying false-positives and also threats that may have been missed, and interpreting and understanding the network-security state [4] - also referred to as the security posture of the organisation being monitored. The human practitioner is crucial to the detection and understanding of attacks and anomalous network conditions, and must also interact with automated systems - deciding the validity of, and acting on, the alerts they produce [5].

Security practitioners involved in monitoring organisational security require tools to help them engage with and understand not only the outputs of automated threat detection tools, but also the raw network data via a range of data presentation techniques. Text-based interfaces and security visualizations are widely used in SOCs to present information about the data travelling through the network, in conjunction with the outputs generated by automated systems $[6,7]$. In this work, we aim to explore the viability of using sonification ("the use of non-speech audio to convey information" [8]) to present security-monitoring data to humans. Our study contributes evidence towards the wider question of the utility of presenting sonified data for use alongside existing tools in SOCs, to aid security practitioners in their monitoring work.

Sonification has been shown to have advantages for presenting data to humans in other fields, such as Medicine and Astronomy, for monitoring data and anomaly detection [9]. In theory, the properties that sonification has been shown to afford in other fields suggest its promise for aiding in SOC working practice, if used alongside the range of tools currently used in SOCs [10]. Sound can be comprehended peripherally, enabling monitoring as a non-primary task [11], which may aid busy SOC analysts. Furthermore humans have strong patternrecognition capabilities using sound [12]. Based on the challenges faced by SOCs, the properties that sonification can afford, and interviews with security practitioners working in SOCs, it has been reported that sonified presentations of network-security information have the potential to aid in SOC working practice [13]. In particular, security practitioners monitoring network security as a primary or non-primary task might benefit from using sonification in tasks such as maintaining situational awareness, detecting anomalous network traffic conditions, and contextualising and triaging the events generated by automated security tools.

On this basis, it is important to assess the extent to which sonification systems can feasibly be used by humans to receive information pertaining to network security. Prior work indicates the potential of network-traffic sonification systems for signalling network attacks $[14,15,16,17]$ but does not experimentally assess their effectiveness. It is this gap in knowledge that we seek to address by assessing the short-term effectiveness of a network-data sonification system 
for signalling attack conditions. More specifically, we assess the viability of using sonification as an approach to enabling humans to detect anomalous conditions in the network traffic by hearing deviations in the sounds representing this traffic.

This paper reports on the results of an effectiveness study we ran with a sonification system, to assess the extent to which participants listening to sonified network datasets could detect and identify network attacks. The sonification system was designed and implemented by ourselves, and sonifies network packet headers, based on a parameter-mapping sonification model. The packet-header data is then presented to humans in this sonic form. The effectiveness of the sonification system was assessed in terms of the capabilities it afforded users: their accuracy and efficiency in attack detection, and their accuracy in attack identification. By detection, we refer to the recognition of some network attack; by identification, an understanding of the attack type signalled.

Our contribution is empirical evidence of the viability of presenting sonified network traffic as an approach to enabling humans to quickly recognise abnormal network conditions, and understand those conditions. We show that a set of network attacks, including attacks occurring simultaneously, could be detected accurately and efficiently by humans listening to the sonification, and that the type of attack could be identified accurately. We also show that musical experience had no significant effect on the ability of participants to use the sonification, and that participants were able to detect attacks as deviations in sound prior to training (with no knowledge of the attacks represented or their mapping to sound).

These results show that attack-detection by humans listening to sonified network traffic is possible, and represent an initial indication of the potential for sonification to represent network-security monitoring information in SOCs. We recognise that there are challenges remaining and highlight these as areas for future work. This includes questions pertaining to the viability of monitoring sonification as a non-primary task in SOCs, the integration of sonification systems with existing SOC tools and soundscapes, and assessment of the potential fatiguing effects of continuous exposure to sonification, for example.

\subsection{Contributions}

Below, we list the contributions we make to the cybersecurity analytics and sonification research communities in this paper.

- We present a novel system for the sonification of network packet headers. The system was developed according to a previously developed high-level model for the sonification of network-security data [10], but the system presented here is novel insofar as it has not been presented previously.

- We present empirical evidence that humans listening to the sonification of network packet headers are able to accurately and efficiently detect, and accurately identify the nature of, network attacks. We consider this an important contribution to cybersecurity analytics and sonification research, 
given that there has been limited evidence supporting the effectiveness of sonification as an approach to "hearing" attacks in network data, as we show in the "Related Work" section.

- We show that humans listening to the sonified packet headers can hear attacks "intuitively" as deviations from the "normal" sound of the background traffic, before training to use the system, and that the accuracy with which humans detected and identified attacks improved over time spent using the system.

- We show that humans can recognise sonified conditions in which two attacks occur simultaneously, and identify the type of these attacks.

- We show that the prior musical experience of a human does not significantly affect their ability to use the sonification system to detect and identify attacks.

\subsection{Structure}

In Section 2, we present relevant related work in sonification for networkattack detection. In Section 3, we present the sonification system, and the network and attack models used for the study. The study hypotheses are detailed in Section 4, and the methodology described in Section 5. In Section 6 we present the results of the study, and use them to assess our hypotheses. The results are discussed in Section 7, and we conclude in Section 8 with directions for future work.

\section{Related Work}

Recent research in network-attack detection methods includes the investigation of automated anomaly-detection techniques using Statistics and Machine Learning $[18,19]$. Methods of presenting network-security data to humans have also been researched in recent years. This includes advanced visualization methods for complex network-security data [7, 20]. Systems that visualise network traffic can enable humans to recognise patterns and anomalies in network activity [5].

Prior experimental work has shown that sonification is useful for monitoring processes and recognising anomalous patterns in data [9]. This research has spanned a wide range of fields, using complex and time-varying data including medical electroencephalogram (EEG) data, and financial market indices $[9,21$, 22]. Experimental results evidence, for example, the improved performance of scientists in identifying anomalous patterns in astrophysical datasets when using sound, compared to when using visual approaches only [23]. These successful applications in other fields support the potential of sonification as an approach to detecting anomalies in network traffic, which is complex and similarly temporal.

Axon et al. used an online survey and interviews to explore the views of 21 security practitioners working in SOCs on the potential utility of sonification 
to their working practice [13]. In that study, security practitioners listened to a network-sonification prototype and discussed the use of sonification in SOCs, rating a number of use-cases using a Likert scale. The results indicated the potential for sonification to aid in the detection of anomalous conditions and security alerts during monitoring as a primary task, and during monitoring as a non-primary task - when practitioners are multitasking monitoring with other work tasks, or are monitoring whilst away from the SOC, for example. The utility of sonification for actually conveying such network-security information was not experimentally assessed in that study.

The same authors summarised the state-of-the-art in sonification for network monitoring [24]. In that survey, the network data features sonified and attack types targeted by prior sonification systems designed with a view to networkattack detection were presented $[14,25,15,26,27,28,16,29,17]$. In these works, the authors report the ability to hear DoS and port-scanning attacks $[14,16]$, and network conditions such as excessive traffic [15] using sonifications of network traffic. There have been user studies carried out to experimentally assess attackdetection capabilities using network-traffic sonification systems, some of which have suggested the potential of sonification for communicating network-security information $[27,30,31]$. The evidence generated thus far by such studies is limited since only a small number of sonification approaches have been tested, often with limited numbers of participants, and without exploration of related questions such as the effect of musical experience on the performance of users. Contributing further experimental results on attack-detection capabilities using sonification is the aim of our study.

There have been a limited number of user studies carried out with sonification systems for other aspects of network-monitoring tasks. Gopinath [32] conducted a usability study, sonifying a range of events in Snort IDS to signal attacks, with results indicating that sonification may increase user awareness in intrusion detection. Unlike our study, that study used event-based sonification of automatically-detected events (i.e. it presents the alerts output from the system), and did not explore the use of sonification of network traffic for enabling humans to detect attack events themselves. Mancuso et al. [27] tested their system with participants searching a packet capture for "target packets" and reported that participants' performance in detecting these packets was not improved by the introduction of a particular sonification design. This was a particular type of activity involved in attack detection, akin to a search function in text, and so is not addressing the function of attack detection more generally (such as pattern detection, for example, as we consider here).

In general, sonification design approaches fall into three categories [33]. 1) In event-based sonification, discrete sounds represent a defined event - as an alert, for example. 2) Parameter-mapping sonification represents changes in data dimensions as changes in acoustic dimensions. 3) In model-based sonification, a user interacts with a model to receive an acoustic response. Based on recommended approaches to achieving goals using sonification, we select a parameter-mapping approach to sonifying the multi-dimensional and timevarying network traffic data that is the focus of this study [34]. We draw on 
existing models for the parameter-mapping sonification of network data in the system design process described in Section 3 [10].

We conclude that sonification is an evidently effective approach to both exploring and monitoring data, and has been shown to enable listening humans to recognise anomalous patterns. Furthermore, sonification can afford capabilities (peripheral monitoring, pattern recognition) that could help to address some of the challenges faced by security practitioners in SOCs, in theory. While prior work has reported the effectiveness of sonification for signalling network attack types $[14,15,16]$, there is very limited experimental evidence of the attack-detection capabilities of humans listening to sonification. Experimental assessment of the use of sonification for signalling network attacks is key to advancing knowledge in this field, and understanding the potential of this approach to network-security monitoring. We posit that the results of this study will provide important insight into the promise of sonification for signalling network-security information, and reflect on the study results from this perspective in Section 7.

\section{Sonification System}

\subsection{System Design}

We chose to use parameter-mapping sonification, in which changes in some data parameters are represented by changes in acoustic parameters; data parameters are mapped to acoustic parameters continuously. A common approach is the mapping of various parameters of sound (pitch, timbre, tempo, for example) to the various dimensions of a multidimensional dataset (a dataset could list types of car, for example, with parameters describing their size, speed and colour). Parameter-mapping sonification has previously been used successfully to create continuous sonifications of datasets with multiple parameters [35, 36]; we therefore elected to apply this sonification approach to continuously represent packet headers, for which we wished to represent multiple parameters (such as the packet size, protocol and source IP, amongst others).

The system was designed according to an existing sonification design model for network data presented by Axon et al. [10]. In that model, an approach to network-data sonification is described in terms of relations between the components: data channels, data dimensions, sound channels and sound dimensions. In Table 1, we briefly describe these components and relations, and show how we used the model to design the sonification system for this study. The mappings from data to sound are described as mathematical relations between data and sound components. For example, the mapping from the discrete data dimension "direction of traffic" $\left(d d \beta_{1}\right)$ to the continuous sound dimension "pan" $\left(d s \alpha_{2}\right)$ is described by the discrete dimension relation: $\left.d d \beta_{1} \rightarrow d s \alpha_{2}\right)$. For details of the model, we refer the reader to the original paper [10]. 
Table 1: Sonification System Design and Basis in Sonification Model [10]. Dark grey bands indicate the relation between data channels and sound channels (between CD and CS), and data dimensions and sound dimensions (between DD and DS), according to the sonification model [10]. Light grey bands indicate the breakdown of data and sound dimensions into continuous and discrete dimensions (according to the sonification model [10]). White bands indicate the data channels and dimensions, and sound channels and dimensions, we selected to create this sonification system.

\begin{tabular}{|c|c|c|c|}
\hline $\begin{array}{l}\text { Data Compo- } \\
\text { nent }\end{array}$ & Relation & $\begin{array}{l}\text { Sound Compo- } \\
\text { nent }\end{array}$ & $\begin{array}{l}\text { Description of Map- } \\
\text { ping }\end{array}$ \\
\hline $\begin{array}{l}\text { Data channels } \\
\text { (CD) (parts } \\
\text { of the data } \\
\text { about which } \\
\text { information is } \\
\text { presented) }\end{array}$ & $\begin{array}{l}\text { Channel } \\
\text { Relation } \\
\left(\operatorname{Rel}_{c}\right)\end{array}$ & $\begin{array}{l}\text { Sound channels } \\
(C S) \text { (streams } \\
\text { of sound which } \\
\text { can be varied } \\
\text { sonically) }\end{array}$ & \\
\hline $\begin{array}{l}\text { Network packet } \\
\left(c d_{1}\right)\end{array}$ & & Note event $\left(c s_{1}\right)$ & $\begin{array}{l}\text { Sampled packet } \rightarrow \text { note } \\
\text { event }\end{array}$ \\
\hline $\begin{array}{l}\text { Data dimen- } \\
\text { sions (DD) } \\
\text { (information } \\
\text { presented } \\
\text { about data } \\
\text { channels) }\end{array}$ & $\begin{array}{l}\text { Dimension } \\
\text { Relation } \\
\left(\operatorname{Rel}_{d}\right)\end{array}$ & $\begin{array}{l}\text { Sound dimen- } \\
\text { sions }(D S) \text { (types } \\
\text { of sonic varia- } \\
\text { tions made to } \\
\text { sound channels) }\end{array}$ & \\
\hline $\begin{array}{l}\text { Data dimen- } \\
\text { sions (continu- } \\
\text { ous) }(D D \alpha)\end{array}$ & $\begin{array}{l}\text { Continuous } \\
\text { Dimen- } \\
\text { sion } \\
\text { Relation } \\
\left(\operatorname{Rel}_{d \alpha}\right)\end{array}$ & $\begin{array}{l}\text { Sound dimen- } \\
\text { sions (continu- } \\
\text { ous) }(D S \alpha)\end{array}$ & \\
\hline $\begin{array}{ll}\text { Packet } & \text { rate } \\
\left(d d \alpha_{1}\right) & \end{array}$ & & Tempo $\left(d s \alpha_{1}\right)$ & $\begin{array}{l}\text { Higher packet rate } \rightarrow \\
\text { faster tempo }\end{array}$ \\
\hline $\begin{array}{l}\text { Packet } \quad \text { size } \\
\left(d d \alpha_{2}\right)\end{array}$ & & $\begin{array}{ll}\text { Note } & \text { duration } \\
\left(d s \alpha_{2}\right) & \end{array}$ & $\begin{array}{l}\text { Longer packet size } \\
\text { longer note duration }\end{array}$ \\
\hline $\begin{array}{l}\text { Data dimen- } \\
\text { sions (discrete) } \\
(D D \beta)\end{array}$ & $\begin{array}{l}\text { Discrete } \\
\text { Dimen- } \\
\text { sion } \\
\text { Relation } \\
\left(\operatorname{Rel}_{d \beta}\right)\end{array}$ & $\begin{array}{l}\text { Sound dimen- } \\
\text { sions (continuous } \\
\text { or discrete) } \\
(D S \alpha, D S \beta)\end{array}$ & \\
\hline $\begin{array}{l}\text { Direction of } \\
\text { traffic }\left(d d \beta_{1}\right)\end{array}$ & & $\operatorname{Pan}\left(d s \beta_{1}\right)$ & $\begin{array}{l}\text { Incoming traffic } \rightarrow \text { left } \\
\text { pan; outgoing traffic } \rightarrow \\
\text { right pan }\end{array}$ \\
\hline $\begin{array}{l}\text { Type of in- } \\
\text { formation } \\
\text { (IP/port) } \\
\left(d d \beta_{2}\right)\end{array}$ & & $\begin{array}{l}\text { Pitch } \\
\left(d s \beta_{2}\right)\end{array}$ & $\begin{array}{l}\text { Source IPs } \rightarrow \text { low } \\
\text { range; destination IPs } \\
\rightarrow \text { medium range; des- } \\
\text { tination ports } \rightarrow \text { high } \\
\text { range }\end{array}$ \\
\hline $\begin{array}{l}\text { Common-ness } \\
\text { (presence in } \\
\text { hotlist) }\left(d d \beta_{3}\right)\end{array}$ & & $\begin{array}{l}\text { Consonance } \\
\left(d s \beta_{3}\right)\end{array}$ & $\begin{array}{l}\text { IP/port in hotlist } \rightarrow \\
\text { consonant notes (notes } \\
\text { that sound "in the har- } \\
\text { mony"); IP/port not } \\
\text { in hotlist } \rightarrow \text { dissonant } \\
\text { notes }\end{array}$ \\
\hline Protocol $\left(d d \beta_{4}\right)$ & & $\begin{array}{l}\text { Instrument } \\
\left(d s \beta_{4}\right)\end{array}$ & $\begin{array}{l}\text { HTTP traffic } \rightarrow \text { string; } \\
\text { TCP traffic } \rightarrow \text { clarinet; } \\
\text { FTP traffic } \rightarrow \text { piano; } \\
\text { Other } \rightarrow \text { strings }\end{array}$ \\
\hline
\end{tabular}


Since the aim of the system is to sonify network packet headers, the data channels and data dimensions are selected to represent the header fields (these fields are presented in Figure 2). Given a network packet capture, the system reads the capture in pseudo real-time (for clarification of the meaning of pseudo real-time in this context, and of how the system could be used differently to sonify live network traffic in near real-time - with slight delay from data parsing and mapping to sound) - see Section 3.3.1), and plays sound events to represent sampled network packets. The parameters of each sound event are determined by characteristics of the packet it represents, according to the mappings presented in Table 1. The resulting sonification system is illustrated in Fig. 1, which is the diagram with which participants were trained in the study.

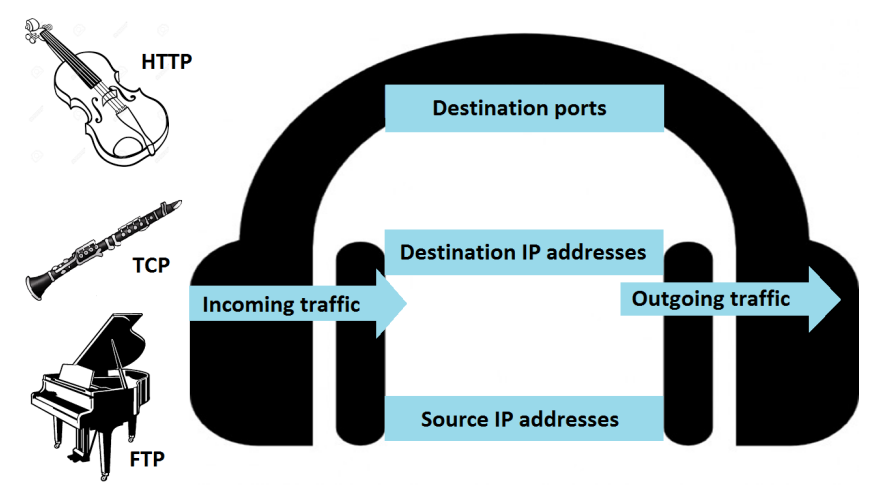

Figure 1: Sonification System Diagram.

The system uses linear sampling of packets, such that the number of sound events played (tempo) correlates linearly with the number of packets observed. A maximum sound event rate is set, to avoid overloading the sound engine. As described in Table 1, the system sonifies incoming and outgoing packet rate, mapping incoming traffic to a left pan (such that all incoming traffic is heard through the left speaker or headphone), and outgoing traffic to a right pan. Internal network traffic is not sonified in the system presented. Selected protocols and applications over which packets are sent are represented by the instruments in which sound events play.

The pitch range within which a sound event plays represents the type of information described. Information about source and destination IPs, and destination ports is played as low- medium- and high-range sound events respectively. For each of these three information types, hotlists (lists of the most commonlyobserved) are calculated based on the first section of the dataset used. For each packet sonified, the IP and port values are checked against their respective hotlists. If the value is found in the hotlist (and is therefore "common"), it is mapped to a consonant note (a note that sounds "in the harmony"); otherwise (if the value is "unusual"), it is mapped to a random dissonant frequency within the pitch range. As such, packets with unusual source IP addresses are represented as three-note chord sound events in which the lowest note (representing 
source IP) is dissonant, for example. The sampling of packets is weighted, such that packets for which the IPs and ports do not fall within their respective hotlists are sampled with a higher probability.

The duration of each note is calculated based on the size of the packet it represents (according to the mapping described in Table 1), such that packets with larger size are represented by a note with longer duration, while small packets are represented by a note short in duration. The maximum duration for which a single note played in the sonification was 1 second.

\subsection{Network-Attack Datasets}

We generated synthetic network-attack datasets to be sonified for the study, based on a network model and a set of attack models. Using synthetic generation enabled us to select the attacks to be experimented with, and produce labelled datasets containing the modelled attacks in an order pseudorandomised for each participant. The datasets were generated as packet capture headers in comma-separated value (CSV) format, and included both background traffic and selected attack traffic. An example of the packet-capture CSV files we generated is presented in Figure 2 (which also shows the packet-header field values we generated).

To create the dataset CSV files, we defined the properties of the background traffic and of the attack traffic. We then used a Python script to populate the CSV files with background traffic probabilistically meeting the defined background traffic properties. The script also injected attack traffic that probabilistically met the defined attack traffic properties, occurring between selected timestamps relative to the background traffic. The way in which we defined the properties of the background and attack traffic, and the probabilistic approach we used, are described in the paragraphs below. In order to generate packet captures with field values as realistic as possible, we drew on statistics collected from real network datasets and attack characteristics [37, 38, 39, 40].

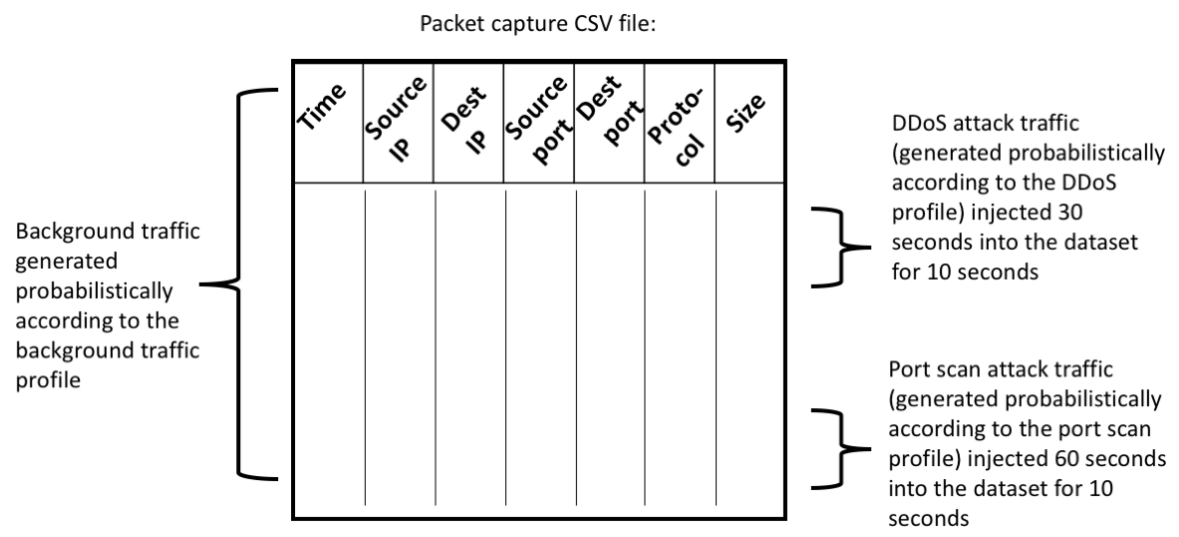

Figure 2: Example of the Method Used to Generate Packet-Capture CSV Files Containing Background and Network-Attack Traffic. 
To define the properties of the background traffic (as indicated in Figure 2 ), we modelled a network in which 25 desktop hosts communicated with 40 remote hosts. The distribution of protocols and applications used was based on statistics collected from existing network datasets [38, 41], by observing average traffic rates for a range of different services. Standard ports were used for the protocols and applications modelled. To imitate network traffic rate distributions, we generated activity for each service with a probabilistic approach using an Interrupted Poisson Process (IPP), in which each network service has two states - on or off [42]. This meant that services were active at any time with a given probability. The background traffic had an average rate of 4500 packets per second. This was scaled up from an average packet rate we derived across the datasets presented in [40] (1750 packets per second) to test whether the sonification could handle realistically-large traffic rates by packet sampling. While some large organisational networks are likely to have higher traffic rates than this, we consider that the traffic level used in this study is valid, due to its basis in traffic levels from real network captures.

We selected four attack types for the study. In selecting the attack types, we considered attacks that could be signalled through the packet information sonified by our system (for example, this system does not sonify packet contents, and we therefore excluded attacks signalled through packet contents only, such as delivery of certain malwares). This list of attacks used is not exhaustive; the aim of this study is to test the concept that attacks can be signalled to humans using sonification, and we therefore selected a subset of the possible attacks. We leave to future work assessment of the full range of attacks that sonification systems are capable of representing. The attacks selected were modelled with the following profiles (which were used to create the attack traffic shown in Figure 2).

- DDoS attack: TCP SYN flood, in which 10 DDoS bots each sent on average 2 TCP SYN packets per second. Compared with the peak DDoS packet rate calculated in the real-world CAIDA DDoS dataset [43] $(175,010$ packets per second), we consider this a small attack.

- Port scan: TCP Connect scan, based on TCP Connect send/respond characteristics [39]. Two scan bots scanned network host ports in a pseudorandom order, each sending on average 15 TCP Connect packets per second.

- Data exfiltration: data exfiltrated over FTP, in which on average 3 FTP packets per second were sent from an internal network source IP address to a single external destination IP address. We drew on a flowlevel breakdown of an FTP transfer [44].

- Loss of connectivity: connectivity loss, in which traffic in each service stopped with some probability.

Based on the network and attack properties described, packet-capture CSVs were generated (as indicated in Figure 2). The packet captures contained back- 
ground traffic (generated according to the defined background traffic properties) and attack traffic injected at selected timestamps (for example, if DDoS attack traffic was injected after 30 seconds of background traffic, it met the defined profile of a DDoS attack). For each participant, we generated a new version of each of the following five datasets, for use in the various stages of the study.

- Pre-training dataset - used in the study before training, to test the "intuitiveness" hypothesis (see Section 4)

- 3 training datasets - used in the second part of training, in which participants practised completing the study task

- Post-training dataset - used in the task after training

These five datasets were generated to contain each of the four attacks, in a pseudorandomised order (using the Random module in Python). As well as the pseudorandomisation of attack orders, there was some variation in the traffic contained in the datasets used for each participant. This was because while the background and attack traffic were generated according to the defined profiles, a probabilistic approach (IPP, as described above) was used to generate the traffic. This variation was intended to produce enough similarity to enable comparison of participants' performances across datasets, and avoid presenting participants with exactly the same dataset each time. We judged that this would enable validation of the effectiveness of the sonification approach, rather than of the participants' ability to learn the exact sounds of the study.

\subsection{Implementation}

We applied the sonification system described in Section 3.1 to sonify the network packet-header CSV files generated as described in Section 3.2. The system reads through the packet headers in the CSV file in pseudo real-time (i.e., it sonified each sampled packet represented in the CSV file at the time at which it occurred, relative to the beginning of the packet capture).

In Figure 3, we illustrate how each sampled packet header was converted to a note event in the sonification, and how the sonification process applied to each individual packet header resulted in a continuous soundscape composed of individual note events. As shown, given the header of an individual packet, the sonification approach generates a note event. The time at which the note event plays is dependent on the timestamp of the packet; the source and destination IP and port values inform the pitch range and the consonance of the pitch of the note, as described in Table 1; the protocol the packet uses informs the instrument in which the note is played, and the size of the packet informs the duration of time for which the note is held. The combination of multiple single note events, generated individually to represent multiple packets, results in a continuous sonification soundscape.

We pre-recorded these sonifications of all datasets in Waveform Audio File (WAV) format, a file format for storing an audio bitstream on PCs, to allow for playing and pausing as necessary by the researcher during the study. These 


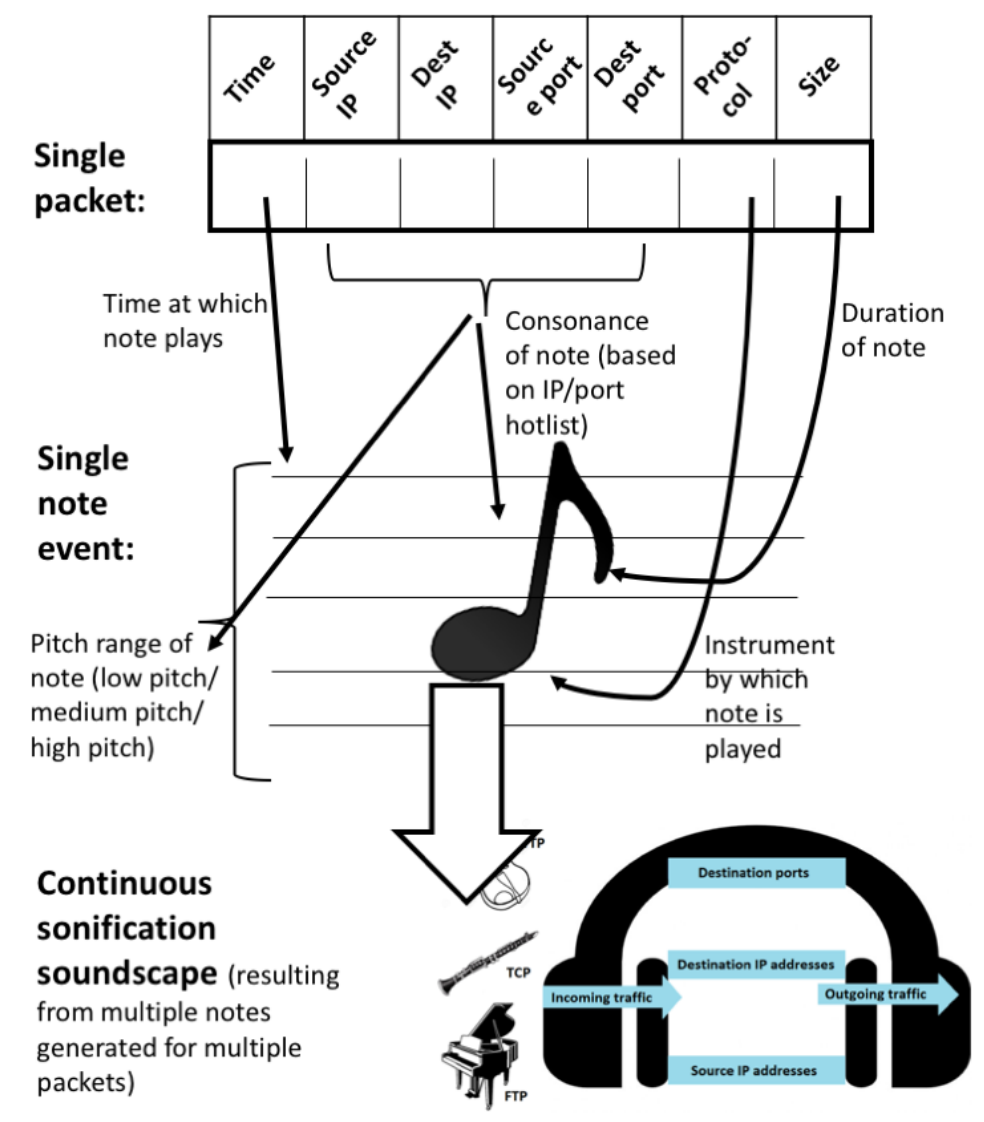

Figure 3: Sonification of Single Packets as Single Note Events, Resulting in a Continuous Sonification Soundscape.

WAV recordings were made using Supercollider, the audio synthesis platform we used as our sonification engine [45]. Figure 4 shows the technical stages involved in converting the packet-capture CSV files to sonification WAV files. As shown, we used a parser written in Python to convert each network-attack dataset CSV into a CSV of sonification values for each packet, ordered by timestamp. We then used a separate Python program to read this parsed output in pseudo real-time, sample packets, and send Open Sound Control (OSC) messages containing the sonification values of the packets sampled to the sonification engine in SuperCollider audio synthesis platform. Fig. 4 illustrates this process.

Given the design of the sonification system, the four attacks chosen for the study sounded as illustrated in Fig. $5^{1}$. Each type of attack had a distinct

\footnotetext{
${ }^{1}$ An example sound clip of a network-attack dataset sonification can be found at https: //soundcloud.com/user-71482294/socs-interview-network-sonification. The times at
} 


\begin{tabular}{|c|c|c|c|c|c|}
\hline \multirow{3}{*}{$\begin{array}{l}\text { CSV file (packet } \\
\text { capture } \\
\text { containing } \\
\text { background } \\
\text { traffic and } \\
\text { network attacks) }\end{array}$} & \multirow{2}{*}{$\begin{array}{l}\text { Python parser } \\
\text { converts } \\
\text { packets to } \\
\text { their } \\
\text { sonification } \\
\text { values }\end{array}$} & \multirow{3}{*}{$\begin{array}{l}\text { CSV file } \\
\text { (sonification } \\
\text { values calculated } \\
\text { for each packet } \\
\text { from the packet } \\
\text { capture CSV) }\end{array}$} & \multirow[b]{2}{*}{$\begin{array}{l}\text { Python Open Sound } \\
\text { Control (OSC) sender } \\
\text { sends sonification } \\
\text { values to } \\
\text { sonification engine }\end{array}$} & \multirow{3}{*}{$\begin{array}{l}\text { Supercollider } \\
\text { sonification engine } \\
\text { converts OSC } \\
\text { messages to sound } \\
\text { (i.e., plays the } \\
\text { sonification) }\end{array}$} & \multirow{3}{*}{$\begin{array}{l} \\
\text { Sonification } \\
\text { (Recorded as } \\
\text { WAV file) }\end{array}$} \\
\hline & & & & & \\
\hline & & & & & \\
\hline
\end{tabular}

Figure 4: Technical Process: Converting Network-Attack CSV Files to Sonification WAV Files.

sound, resulting from the sonification mappings used (as presented in Table 1) and the characteristics of the attack (as presented in Section 3.2). As shown in Figure 5, the DDoS attack, in which an increase in incoming traffic from unusual (non-hotlisted) source IP addresses (bots) is seen, can be heard as an increase in the amount of dissonant activity in a low pitch range (since an increased number of packets are observed from source IP addresses that are not hotlisted, and these packets are sonified as dissonant notes in a low pitch range - according to the sonification mappings in Table 1). Since the DDoS modelled is a TCP SYN flood, the attack is heard in the clarinet in this instance (since TCP packets are represented in the clarinet, as shown in Table 1). For the port scan, we hear a range of unusual destination ports being targeted as an increase in dissonant activity in a high pitch range (since destination ports are represented in the high pitch range by the sonification system). We hear data being exfiltrated over FTP as an increase in the piano activity with a right pan, to an unusual destination IP address (a dissonant medium-range note), since FTP traffic is represented in the piano, and outgoing traffic is panned to the right. The loss of connectivity fades to silence as activity comes to a stop in each service and therefore no packets are observed and sonified.

\subsubsection{Note on Real-Time Implementation}

Throughout this paper, we refer to sonification of network-traffic as being in "pseudo real-time". Here, we refer to the fact that the sonification system plays through a CSV file, creating sound events at the time at which traffic events occur (relative to the beginning of the CSV file). In this paper we have not actually implemented the sonification system in real-time: we generated the CSV files of packet captures in advance (i.e., they do not represent network traffic captured in real-time), and also generated and recorded the sonifications of this traffic in advance. These decisions were taken to reduce the risk of errors occurring during the study. We posit that the system we present could be used to enable near real-time sonification of live network traffic, however.

In Figure 6 we present an adaptation of the technical process captured in Figure 4, to show how this process would be adapted for near real-time sonification (slight delays resulting from data parsing and the mapping of data values to

which the different attacks occur in the sound clip are described at this URL. 

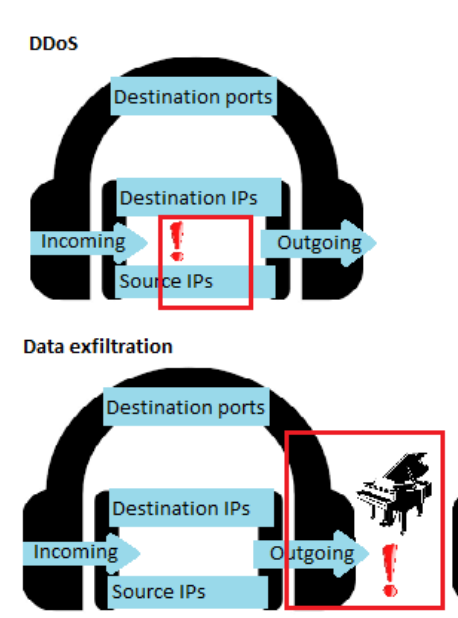

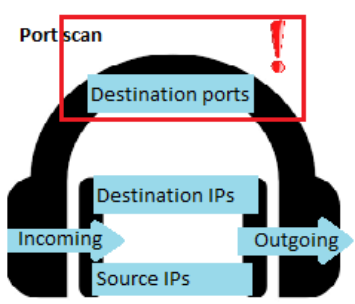

Loss of connectivity

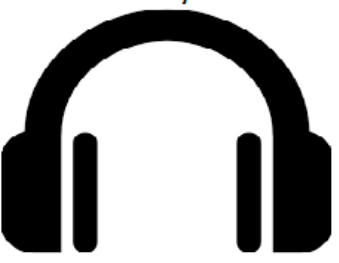

Figure 5: Representations of Network Attacks in the Sonification.

sound prevent it from being in real-time). Rather than storing packets as CSV files in advance of the sonification process, in this near real-time approach, as each individual packet is captured live on the network, it is parsed to produce sonification values, which are then sent as OSC messages to the sonification engine. This means that humans listen to the sonified packets as the packets are observed on the network, rather than listening to a pre-recorded WAV file as in this study.

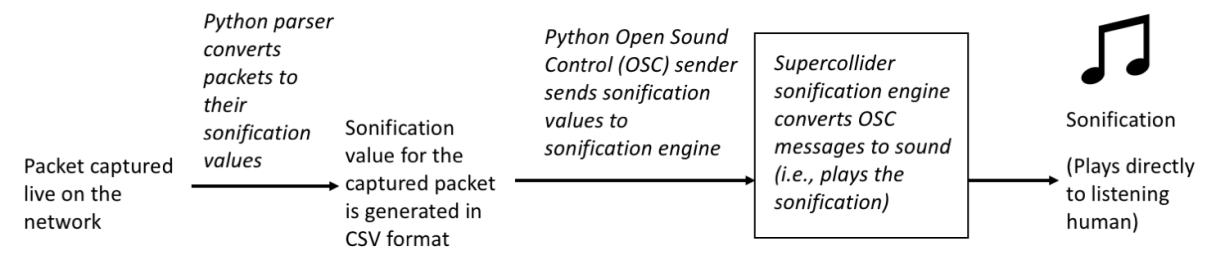

Figure 6: Technical Process: How Live Network Packets Could be Converted to Near RealTime Sonification.

\section{Study Motivation, Research Questions, and Hypotheses}

The motivation for this study was to assess the accuracy and efficiency with which participants could detect the presence of an attack in the sonification, and the accuracy with which they could identify the type of attack detected. We differentiate between detection and identification of attacks: detection refers to the recognition that some attack is being signalled by the sonification, without necessarily knowing the type of attack; identification means understanding the type of attack signalled. 


\subsection{Research Questions}

In line with the above motivation, the main research question we aimed to address was the following. This main research question was broken down into seven component research questions. For each of these seven questions, we developed a hypothesis to be assessed, resulting in the seven hypotheses we present in Section 4.2 (the hypothesis developed to address each research question is referenced at the side of the relevant research question).

Main research question: how effectively can the developed sonification system, when applied to network packet-header datasets containing attacks, enable humans to hear the attacks present?

1. How accurately can humans detect the occurrence of an attack by listening to the sonified packet headers? (leads to Hypothesis A)

2. How efficiently (quickly) can humans detect occurrence of an attack by listening to the sonified packet headers? (leads to Hypothesis B)

3. Having detected an attack occurring, how accurately can humans identify the nature or type of the attack by listening to the sonified packet headers? (leads to Hypothesis C)

4. Can humans recognise the occurrence of multiple attacks in combination by listening to the sonified packet headers, and can they identify the types of these attacks? (leads to Hypothesis D)

5. Can humans detect attack conditions intuitively, before having been trained to use the sonification approach, by listening for "abnormal" sounds compared with a "normal" baseline traffic sound? (leads to Hypothesis E)

6. How does musical experience affect the ability of participants to use the sonification system to detect and identify attacks? (leads to Hypothesis F)

7. How does familiarity with the sonification system (training and length of time spent using it) affect the ability of participants to use the sonification system to detect and identify attacks? (leads to Hypothesis G)

Aside from these performance-based questions (which focus on how well users of the sonification system perform in attack-detection tasks), there remain wider questions relevant to the utility of sonification systems for network-attack detection. Exploring the usability of sonification systems in this use-case (including answering questions around the extent to which listening to sonification systems causes fatigue) will be important to understanding the utility of the attack-detection approach. While such questions fell outside the scope studied here, we highlight them as key areas for future work in Section 8. 


\subsection{Study Hypotheses}

Hypothesis A: Accurate Attack Detection

We hypothesised that network attacks could be detected accurately by listening to the sonification. It is important that users can detect the presence of an attack in the sonification with a level of accuracy: that attacks are detected (high true-positive rate) and not missed (low false-negative rate), and that the sonification does not signal attacks to listeners when attacks are not present in the network data (low false-positive rate). We assessed this hypothesis by measuring the number of true-positive, false-positive and false-negative attack detections by participants (the method of measurement for these hypotheses is described in Section 5.2) and using these measurements to calculate precision, recall and F-score [46] for the detection of attacks overall, and for each attack type presented (wherever we use the term "F-score" in this paper we refer to the harmonic average of precision and recall [46], as defined in Section 6, rather than to the ANOVA F-statistic). In the post-training task, we consider the minimum acceptable F-score to be 0.9 , a value which indicates a reasonably high level of accuracy similar to that attained in other attack-detection research $[47,2]$.

\section{Hypothesis B: Efficient Attack Detection}

We hypothesised that network attacks could be detected efficiently (within a short time after the beginning of an attack) by listening to the sonification. For the timely detection of network attacks, it is important that these conditions are signalled by the sonification in a way that enables humans to detect them quickly. To assess the detection efficiency for each attack type individually, and across all attacks, we measured the time between the beginning of an attack in the dataset sonified, and the first subsequent true-positive detection of that attack by the participant. We aimed to test the following: for each attack type $A, \Delta t_{A}<\operatorname{tmax}_{A}$, where $\operatorname{tmax}_{A}$ is the maximum time we consider acceptable for detection of attack $A, \Delta t_{A}=$ is the mean average across all $k$ participants of the difference $\delta t_{A k}=t d_{A k}-t b_{A k}$, between the time of the beginning of attack type $A, t b_{A k}$, and the time of its detection $t d_{A k}$. For this study, we set $\operatorname{tmax}_{A}$ to be 3 seconds. We consider that detection of attacks less than 3 seconds after their beginning is acceptable, 3 seconds being a reasonable estimate of the length of time within which humans working in SOCs could usually be expected to be made aware of alerts produced by automated systems, or of network anomalies by observing the data itself.

\section{Hypothesis C: Accurate Attack Identification}

We hypothesised that network attack types could be identified accurately (that the participant could correctly specify the attack type) by listening to the sonification, after training. The aim was to explore the extent to which participants could glean information from the sonification, so that rather than recognising only that some attack is occurring, as tested in Hypothesis A, they were able to accurately identify the type of attack they had heard. We assessed, for each attack accurately detected, whether the participant was able to accurately describe its type. 
Hypothesis D: Combined Attacks

We hypothesised that participants could recognise when two attacks occur simultaneously, and identify the attack types. In reality, networks may be hit by multiple attacks simultaneously. This might mean the coincidence of two unrelated attacks from different sources, or might mean the use of one attack (a DDoS, for example) to distract attention from another (such as malware or exfiltration of data). It is, therefore, important to assess the potential for sonification to signal combined attack conditions.

\section{Hypothesis E: Intuitiveness of Attack Detection}

We hypothesised that participants could hear "normal" and "abnormal" sounds (where "normal" and "abnormal" sounds were not defined to participants but were left for participants to judge - see Section 5.1.1) representing attacks intuitively before training, but would detect attacks more accurately and efficiently after training. Once trained to use the sonification and detect the study attack types used, participants should know which sounds to expect. It is important to assess whether the sonification signals abnormal conditions related to attacks intuitively, before users have a knowledge of the sounds of expected attack types.

The motivation for testing whether participants could hear abnormal network conditions without training, was to explore the potential for sonification to represent unknown, or variations on known, attack types (as in these cases, users would not be listening for "expected" attack sounds). We aimed to test the following for pre-training dataset $d_{1}$ and post-training dataset $d_{4}$ : $\operatorname{amin}_{d 1}<a_{d 1}<a_{d 4}$ and emin $e_{d 1}<e_{d 1}<e_{d 4}$, where $a_{d n}, e_{d n}, n \in(1,4)$ are respectively the accuracy and efficiency of attack detection of participants in the $n^{\text {th }}$ dataset, and $\operatorname{amin}_{d 1}, \operatorname{emin}_{d 1}$ are respectively the minimum accuracy and efficiency we accept in dataset $d_{1}$. We consider the minimum acceptable F-score $\left(\operatorname{amin}_{d 1}\right)$ in the pre-training task to be 0.7 , given that participants have no knowledge of the sounds of the attacks at this stage. As explained in Hypothesis $\mathrm{B}$, we considered a 3 -second average across participants to be the minimum attack-detection efficiency acceptable $\left(\operatorname{emin}_{d 1}\right)$.

\section{Hypothesis F: The Effect of Musical Experience}

We hypothesised that a participant's level of musical experience would not affect his ability to detect and identify attacks using the sonification. For the integration of sonification into SOCs, and its use by security analysts, it is important that the approach does not favour users who are musically-experienced, and that users without musical experience are equally able to detect and identify network attacks. We aim to test the following: $a_{m u s}=a_{\text {nonmus }}, e_{m u s}=e_{\text {nonmus }}$, and $i_{m u s}=i_{\text {nonmus }}$, where $a_{m u s}$ and $a_{\text {nonmus }}$ are the detection accuracy, $e_{m u s}$

and $e_{\text {nonmus }}$ the detection efficiency, and $i_{\text {mus }}$ and $i_{\text {nonmus }}$ the identification accuracy of participants with and without musical experience respectively. 
Hypothesis G: Improvement Through Familiarity

We hypothesised that participants' accuracy and efficiency in detecting attacks would improve with time spent using the sonification. It is beneficial if users can improve at, and become comfortable with, using the sonification over time, detecting and identifying attacks more accurately and quickly. If this is the case, we argue that sonification is a network-monitoring approach that users can potentially come accustomed to, continuously improving their understanding of its signals. For this study, we aimed to measure participants' performance at each stage of use of the sonification, and assess whether there was an improvement over time. Formally, we aimed to test the following hypotheses over the pre-training dataset $\left(d_{1}\right)$, training datasets 2 and $3\left(d_{2}\right.$ and $d_{3}$ respectively), and post-training dataset $\left(d_{4}\right): a_{d 1}<a_{d 2}<a_{d 3}<a_{d 4}, e_{d 1}<e_{d 2}<e_{d 3}<e_{d 4}$, and $i_{d 1}<i_{d 2}<i_{d 3}<i_{d 4}$, where $a_{d n}, e_{d n}$, and $i_{d n}, n \in(1, . ., 4)$ are respectively the accuracy of attack detection, efficiency of attack detection, and accuracy of attack identification of participants in the $n^{\text {th }}$ dataset.

\section{Methodology}

\subsection{Study Process}

The study was carried out with 30 computer science and cybersecurity researchers at the University of Oxford. Ethical approval for this study was given by the University of Oxford Central University Research Ethics Committee. Prior to the training and study tasks, participants were asked to answer some preliminary demographic questions, including questions on hearing disabilities. Participants were also asked to indicate their level of musical training. In the study, participants sat with their backs to a monitor, facing the researcher across a table. On the monitor, the "testing webpage" was presented, using which the researcher presented the audio clips to participants, and recorded the mouse clicks made by participants. More information on this webpage is given in Section 5.2.

Participants were positioned at an equidistance between two speakers (the left and right speaker, see pan mapping), with a computer mouse positioned in front of them, as illustrated in Fig. 7. They then completed the following stages of the study. 


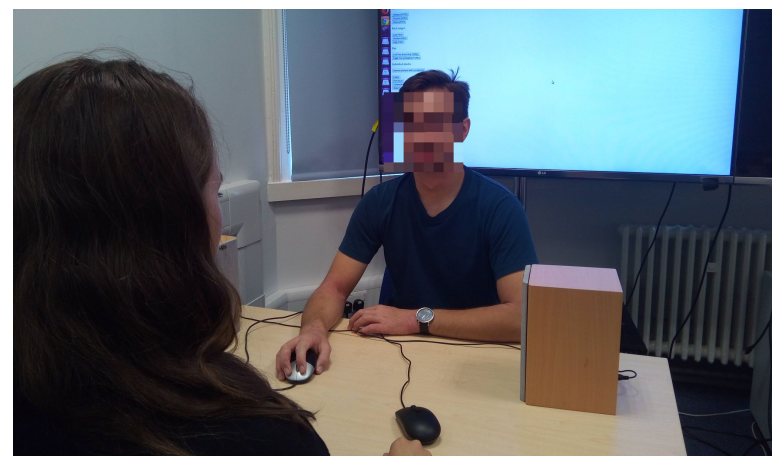

Figure 7: Study setup. Participants sat with their backs to the monitor presenting the "testing webpage" (from which the researcher presented the audio clips), with a computer mouse in front of them, facing the researcher, at an equidistance between the two speakers.

Table 2: Hypotheses Tested at Each Stage of the Study

\begin{tabular}{lll}
\hline Stage & Measurements taken & $\begin{array}{l}\text { Hypotheses } \\
\text { tested }\end{array}$ \\
\hline $\begin{array}{l}\text { Pre-training } \\
\text { task }\end{array}$ & Attack detection: accuracy, efficiency & $\mathrm{A}, \mathrm{B}, \mathrm{E}, \mathrm{F}, \mathrm{G}$ \\
$\begin{array}{l}\text { Training } \\
\text { dataset } 2\end{array}$ & Attack detection: accuracy, efficiency & $\mathrm{A}, \mathrm{B}, \mathrm{F}, \mathrm{G}$ \\
\hline $\begin{array}{l}\text { Training } \\
\text { dataset } 3\end{array}$ & $\begin{array}{l}\text { Attack detection: accuracy, efficiency } \\
\text { Attack identification: accuracy }\end{array}$ & $\mathrm{A}, \mathrm{B}, \mathrm{C}, \mathrm{F}, \mathrm{G}$ \\
\hline $\begin{array}{l}\text { Post-training } \\
\text { task }\end{array}$ & $\begin{array}{l}\text { Attack detection: accuracy, efficiency } \\
\text { Attack identification: accuracy }\end{array}$ & $\mathrm{A}, \mathrm{B}, \mathrm{C}, \mathrm{F}, \mathrm{G}$ \\
$\begin{array}{l}\text { Combined } \\
\text { attacks task }\end{array}$ & $\begin{array}{l}\text { Combined attack identification: } \\
\text { accuracy }\end{array}$ & $\mathrm{D}$ \\
\end{tabular}

\subsubsection{Pre-training study task}

Participants were played a 30-second sonification of a network dataset containing no attacks - a representation of the "normal sound of the network". They were then played a sonification of a network dataset containing all four attacks in a pseudorandomised order, and were asked to click the mouse button whenever they heard anything "abnormal". The aim was to assess the extent to which the attacks sounded intuitively "abnormal" compared with the usual network sound, and to compare results with the post-training results to assess the improvement of participants. We deliberately did not define "normal" and "abnormal" sounds to participants beforehand, but left this to participants to judge. This was because we aimed to assess (in the pre-study task) whether musical transformation can allow non-attack and attack traffic to sound "normal" and "abnormal", respectively, to people with little definition of what "normal" and "abnormal" are. 


\subsubsection{Training}

In the first stage of training, participants were introduced to the method behind the sonification system, and to the sonification diagram and mappings table (presented in Fig. 1 and Table 1). The design and sounds of the sonification system were described by the researcher, with the individual mappings of the sonification system (such as the instruments, pitch ranges, and left and right pan involved) played individually. The attacks used in the study were then presented in a pseudorandomised order, and their effect on the sonification system described using a second set of diagrams (Fig. 5). Sounds and descriptions were repeated at participants' request.

In the second stage of training, participants were taught to respond to sonified datasets as required in the study task, using three training network-attack dataset sonifications. For the first dataset, participants were asked to listen without responding, and consider the system design and attacks presented. For the second, participants were asked to click the mouse button whenever they heard an attack. For the final training dataset, participants were instructed to click at each attack and subsequently describe verbally the change they heard in the sound and the attack type signalled (this verbal response was prompted by the researcher where necessary).

\subsubsection{Post-training study task}

A sonification of a new network-attack dataset was played. As in the final training dataset, participants were asked to click when they heard an attack, then describe the musical change and the type of attack

\subsubsection{Combined attack task}

Participants were played a short sonification of a network dataset, containing two attacks occurring simultaneously (any pair from DDoS, port scan, and data exfiltration). Instruction in this task was deliberately vague, with the aim of not indicating any information about the contents of the datasets to participants: "you will be played a sonification of a dataset. Please listen, and at the end describe what you think happened in the dataset". The attack pairing presented to each participant was pseudorandomised.

Table 2 illustrates the measurements taken and the hypotheses tested at each stage. Following the study, participants were interviewed about their experience using the sonification, the ease of attack detection and identification, and their view of the training stage and the sonification tool. We manually coded this interview to identify common themes related to the study hypotheses, sonification design, and study design, which we present in Section 7.

\subsection{Measuring Participants' Performance}

Recordings of each of the sonified datasets were made for each participant, and used as input to a series of testing webpages. These webpages were used at each stage of the study (a "pre-training" webpage was used at the pre-training stage of the study, for example), and contained the audio files to be presented 
to the participant at that stage of the study. This webpage was not seen by participants (who sat with their backs to the screen, as shown in Figure 7), and enabled playing and pausing of the audio recordings by the researcher. The webpage also recorded the time at which the participant made mouse clicks in relation to the audio playing, by recording a timestamp (relative to the audio clip playing) each time the mouse was clicked anywhere on the screen. We were thus able to ascertain, for each recording of a sonified dataset, the time during the dataset at which the participant clicked the mouse button having "heard an attack".

In the calculation of detection and identification accuracy and efficiency, we made the assumption that a participant's mouse click was a detection of the attack sonified at that point in the audio. To support this, we left gaps of around 20 seconds between each attack sonified in the study task, and we discuss the implications of this assumption further in Section 7. Using the click timestamps and verbal responses collected, we measured detection accuracy and efficiency, and identification accuracy, as described in Table 3. Since click timestamps were recorded during four different tasks for each participant (pre-training, training dataset 2, training dataset 3 , and post-training task), and the dataset used for each of these tasks presented each of the attack types exactly once, we obtained results on the detection of each attack 4 times per participant. Since there were 30 participants in total, we therefore obtained 120 detection results for each attack type. 
Table 3: Measuring Performance in the Study

\begin{tabular}{|c|c|c|}
\hline $\begin{array}{l}\text { Measurement } \\
\text { taken }\end{array}$ & Method & Assessment criteria \\
\hline $\begin{array}{l}\text { Detection } \\
\text { accuracy }\end{array}$ & $\begin{array}{l}\text { We recorded the time } \\
\text { at which the par- } \\
\text { ticipant clicked the } \\
\text { mouse, and compared } \\
\text { this with the times } \\
\text { between which the at- } \\
\text { tack happened in the } \\
\text { dataset. Mouse clicks } \\
\text { that fell within these } \\
\text { attack times were } \\
\text { marked correct; all } \\
\text { others were marked } \\
\text { incorrect }\end{array}$ & $\begin{array}{l}\text { - If mouse clicked during attack } \\
\text { time window } \rightarrow \text { true-positive } \\
\text { detection } \\
\text { - If mouse clicked outside at- } \\
\text { tack time window } \rightarrow \text { false- } \\
\text { positive detection } \\
\text { - If button not clicked during } \\
\text { attack window (i.e. attack } \\
\text { missed) } \rightarrow \text { false-negative de- } \\
\text { tection }\end{array}$ \\
\hline $\begin{array}{l}\text { Detection effi- } \\
\text { ciency }\end{array}$ & $\begin{array}{l}\text { For all true-positive } \\
\text { attack detections (as- } \\
\text { sessed as described } \\
\text { above), we calculated } \\
\text { the time difference be- } \\
\text { tween the beginning } \\
\text { of the attack and } \\
\text { the participant's first } \\
\text { mouse click following } \\
\text { it }\end{array}$ & $\begin{array}{l}\text { If true-positive detection: } \\
\text { - Time difference between start } \\
\text { of attack and first mouse click } \\
\rightarrow \text { detection efficiency }\end{array}$ \\
\hline $\begin{array}{l}\text { Identification } \\
\text { accuracy }\end{array}$ & $\begin{array}{l}\text { For all true-positive } \\
\text { detections (assessed as } \\
\text { described above), we } \\
\text { marked participants' } \\
\text { verbal description of } \\
\text { the attack type cor- } \\
\text { rect or incorrect }\end{array}$ & $\begin{array}{l}\text { If true-positive detection: } \\
\text { - If attack correctly described } \\
\rightarrow \text { correct identification } \\
\text { - If attack incorrectly described } \\
\rightarrow \text { incorrect identification }\end{array}$ \\
\hline
\end{tabular}

\subsection{Reliability}

Pseudorandomisation of attack order was used throughout the study to avoid bias. The order in which the individual attacks were presented was pseudorandomised for the first part of training. Participants were presented with a pretraining attack dataset, three datasets containing attacks, and a post-training study attack dataset. All datasets were generated individually for each participant, and the order in which attacks appeared in each dataset was pseudorandomised. Finally, participants were presented with a dataset contained a pseudorandomised one of three combinations of two attacks (DDoS/port scan; DDoS/data exfiltration; port scan/data exfiltration). 
Potential biasing factors were eliminated from the study setup as much as possible. Participants sat with their backs to the screen in a deliberately clear room, to avoid visual distractions. The mouse click (anywhere on screen) and verbal response method by which participants were asked to respond to attack detections was intended to be simple, to reduce the time delay introduced by the participants actually carrying out the response method as much as possible.

\section{Results}

Fig. 8 shows the mouse click times of participants in the pre-training task, compared with the attack times. Fig. 9 shows those post-training. In these figures, the height of the bars represents the number of mouse clicks made by participants at that time (indicating that the participant had detected an attack). The green bands indicate the periods of time during which attacks were occurring. Therefore, a high bar at the beginning of a green band indicates that a high number of mouse clicks were made by participants following the beginning of an attack in the dataset.

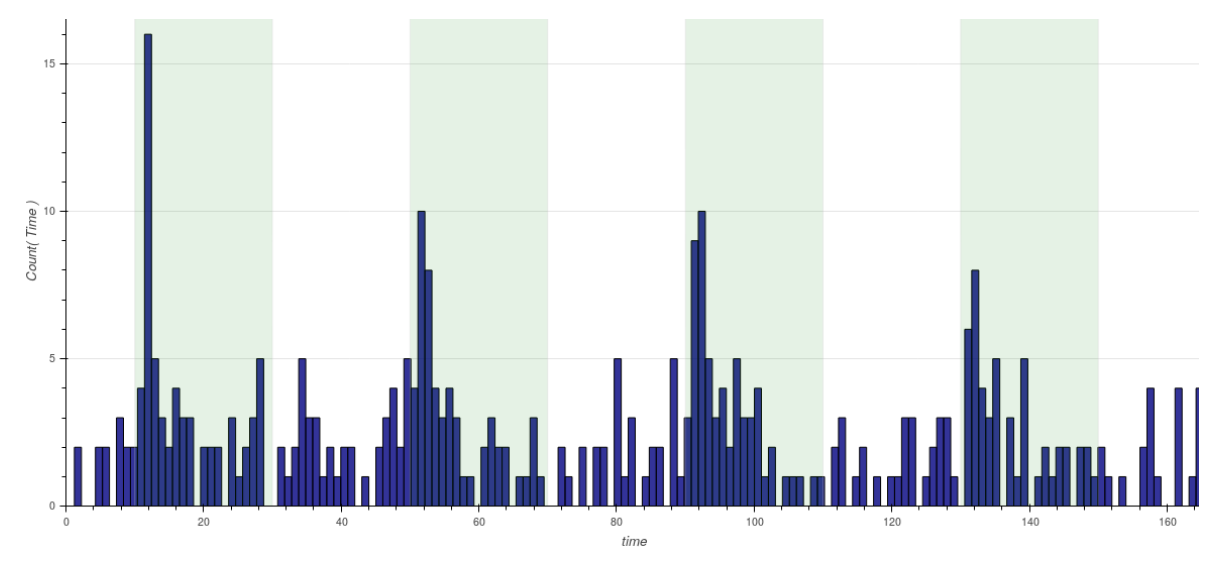

Figure 8: Participant Click Times in Pre-Training Task.

Of the 23 male and 7 female participants in the study, 20 claimed to have some level of musical training (both taught and self-taught), while 10 did not. More specifically, we considered participants to be trained musically if they reported any of the following: some instructed musical training; some self taught musical training; having reached a graded standard in a musical instrument; having advanced (university/conservatoire-level) musical training. All participants claimed either to know the TCP/IP model or to have some theoretical knowledge about network-security monitoring principles. 13 participants reported limited, and 3 extensive, practical experience of network-security monitoring. There were no reported medical hearing disabilities, and all participants reported that their hearing was not currently affected by past experiences (such as loud musical concerts). In Tables 4 - 6 we present the described demographics 


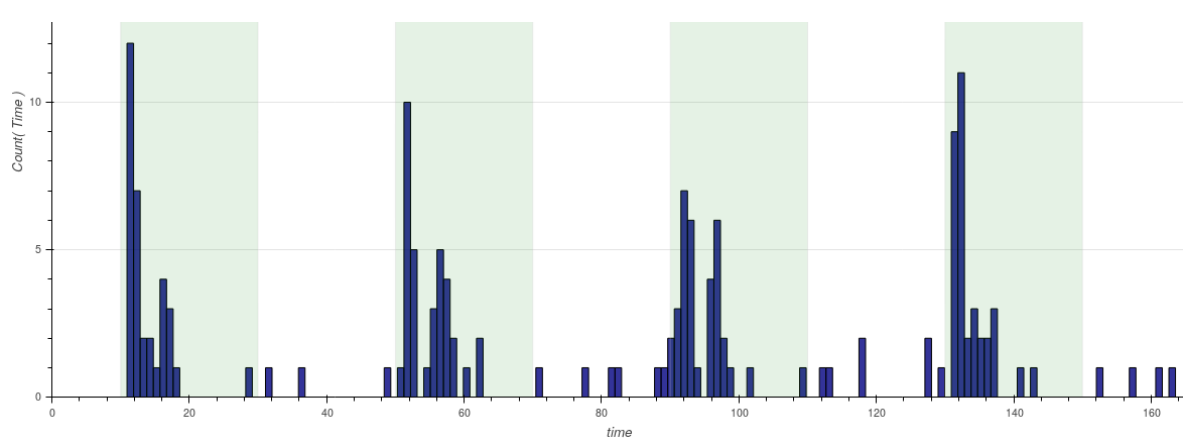

Figure 9: Participant Click Times in Post-Training Task.

of participants: gender, level of musical training, and level of network-security monitoring experience.

Table 4: Participant Demographics: Gender

\begin{tabular}{ll}
\hline Male & Female \\
\hline 23 & 7
\end{tabular}

Table 5: Participants: Level of Musical Experience

\begin{tabular}{l|l}
\hline Level & Number \\
\hline No musical training, does not actively listen to music & 2 \\
\hline No musical training, does actively listen to music & 9 \\
\hline Has some self-taught musical training & 3 \\
\hline Has some instructed musical training & 7 \\
\hline Has reached a graded standard in a musical instrument & 7 \\
\hline $\begin{array}{l}\text { Has advanced (university/ conservatoire-level) musical } \\
\text { training }\end{array}$ & 2 \\
\hline $\begin{array}{l}\text { Has perfect pitch (perfect pitch describes the ability of a per- } \\
\text { son to identify or recreate a given musical note without a } \\
\text { reference tone) }\end{array}$ & 1
\end{tabular}


Table 6: Participants: Level of Network-Security Monitoring Experience

\begin{tabular}{l|l}
\hline Level & Number \\
\hline $\begin{array}{l}\text { Has no network-security monitoring experience and no } \\
\text { knowledge about network-security monitoring }\end{array}$ & 2 \\
\hline $\begin{array}{l}\text { Has some theoretical knowledge about network-security mon- } \\
\text { itoring principles }\end{array}$ & 12 \\
\hline Has some limited practical experience of network-security & 13 \\
monitoring & \\
\hline $\begin{array}{l}\text { Has extensive practical experience of network-security mon- } \\
\text { itoring (having worked as a network-security analyst, for } \\
\text { example) }\end{array}$ & 3 \\
\hline Knows the TCP/IP model & 28
\end{tabular}

Hypothesis A: Accurate attack detection

We used the true-positive $(t p)$, false-positive $(f p)$ and false-negative $(f n)$ detection rates, measured as described in Table 3, to calculate the precision, recall and F-score for participants in the post-training study task. The proportion of true-positive attack detections for all attack types pre- and post-training are shown in Table 7 . The precision, recall and F-score values calculated for all tasks are presented in Table 8.

- Precision $=\frac{t p}{t p+f p}$ takes values in the range $(0,1)$, and is the probability that an object is relevant given that it is returned by the system [46]: here, the probability that an attack was occurring given that the participant clicked.

- Recall $=\frac{t p}{t p+f n}$ takes values in the range $(0,1)$, and is the probability that a relevant object is returned [46]: here, that when an attack occurred, the participant clicked.

- $\mathrm{F}$-score $=2 \frac{\text { precision } * \text { recall }}{\text { precision }+ \text { recall }}$ takes values in the range $(0,1)$, and is the harmonic mean of precision and recall [46] (wherever we use the term "Fscore" in this paper we refer to this harmonic average of precision and recall rather than to the ANOVA F-statistic).

Table 7: Proportion of Participants Who Accurately Detected Each Attack Type Pre- and Post-Training

\begin{tabular}{lllll}
\hline Attack type & $\begin{array}{l}\text { Pre- } \\
\text { training }\end{array}$ & $\begin{array}{l}\text { Training } \\
\mathbf{2}\end{array}$ & $\begin{array}{l}\text { Training } \\
\mathbf{3}\end{array}$ & $\begin{array}{l}\text { Post- } \\
\text { training }\end{array}$ \\
\hline All & $110 / 120$ & $116 / 120$ & $118 / 120$ & $120 / 120$ \\
DDoS & $30 / 30$ & $30 / 30$ & $30 / 30$ & $30 / 30$ \\
Port scan & $30 / 30$ & $30 / 30$ & $30 / 30$ & $30 / 30$ \\
Data exfiltration & $29 / 30$ & $30 / 30$ & $30 / 30$ & $30 / 30$ \\
Loss of connectivity & $21 / 30$ & $26 / 30$ & $28 / 30$ & $30 / 30$
\end{tabular}


Table 8: Precision, Recall and F-score for Pre-Training, Training and Post-Training Datasets

\begin{tabular}{llll}
\hline Dataset & Precision & Recall & F-score \\
\hline Pre-training & 0.61 & 0.96 & 0.75 \\
Training 2 & 0.78 & 0.98 & 0.87 \\
Training 3 & 0.84 & 0.98 & 0.91 \\
Post-training & 0.85 & 1 & 0.92
\end{tabular}

The F-score (0.93) shows that participants were able to detect attacks accurately. The recall score (1) shows that no attacks were missed by participants (no false-negatives) post-training, while the precision (0.86) shows that participants generally clicked at the time of attacks in the dataset - the false-positive detection rate was low.

\section{Hypothesis B: Efficient attack detection}

To assess the efficiency of attack detection post-training, we calculated the mean and standard deviation of the time between the start of each attack and the first mouse click of all participants who accurately detected it. These results are presented in Table 9, which shows mean detection times less than 3 seconds for DDoS, port scan and data exfiltration attacks, and a longer mean detection time for connectivity loss.

Table 9: Post-Training, Efficiency of Attack Detection in Seconds

\begin{tabular}{lll}
\hline Attack type & $\begin{array}{l}\text { Detection time: } \\
\text { mean }\end{array}$ & $\begin{array}{l}\text { Detection time: } \\
\text { standard deviation }\end{array}$ \\
\hline DDoS & 2.43 & 1.64 \\
Port scan & 2.66 & 1.39 \\
Data exfiltration & 2.99 & 1.67 \\
Connectivity loss & 6.44 & 1.82
\end{tabular}

We assessed the variance in efficiency of detection across the four different attack types using one-way Analysis of Variance (ANOVA) analysis [48] with significance $\alpha=0.05$. We obtained $P$-value $<\alpha$, and therefore there is a significant difference in the means across the four attack types. We then discounted the loss of connectivity from this analysis, since clearly the detection time average was much longer (see Table 9). We believe this was due to the study setup and the way the loss of connectivity was represented by the sonification: from the time connectivity loss began in the data, services stopped with some probability, such that the sound thinned but sometimes did not reach a significantly quieter or silent level for 5 or more seconds. This accounts for the much longer detection time for this condition. We performed one-way ANOVA analysis across the remaining three attack types: DDoS, port scan and loss of connectivity. Table 10 shows the results. Since $P$-value $>\alpha$, there is no significant difference in the mean detection times across these three attack types. 
Table 10: Single-Factor ANOVA Analysis of Mean Detection Times for DDoS, Port Scan and Data Exfiltration

\begin{tabular}{lllllll}
\hline Source of Variation & SS & df & MS & F & P-value & F crit \\
\hline Between Groups & 4.75 & 2 & 2.38 & 0.93 & 0.40 & 3.10 \\
Within Groups & 222.23 & 87 & 2.55 & & & \\
& & & & & & \\
Total & 226.99 & 89 & & & &
\end{tabular}

Hypothesis C: Accurate attack identification

To assess identification accuracy, we calculated the proportion of correct identifications for each accurately-detected attack, measured as described in Table 3. These results are presented in Table 11, which shows that 114/120 detected attacks were identified correctly, but port scan was identified incorrectly 6 times. Each of these times, the port scan was identified as a DDoS by the participant. We discuss the possible reasons for this trend in Section 7.3.

Table 11: Post-Training Accuracy of Attack Identification

\begin{tabular}{ll}
\hline Attack Type & $\begin{array}{l}\text { Proportion Accurately Identified (of Those } \\
\text { Detected) }\end{array}$ \\
\hline All attacks & $114 / 120$ \\
DDoS & $30 / 30$ \\
Port scan & $24 / 30$ \\
Data exfiltration & $30 / 30$ \\
Connectivity loss & $30 / 30$
\end{tabular}

Participants were able to accurately identify attacks most of the time - the exception being the confusion for some (6) participants between the sounds of DDoS and port scan. We discuss the implications for sonification design in Section 7. This suggests that sonification is not only a viable approach to detecting abnormalities in the network traffic, but that it also holds promise for signalling information about the attack type.

Hypothesis D: Combined attack identification

We observed three outcomes in this task:

1. participant recognised it was combined and identified both attacks correctly;

2. participant recognised it was combined and identified one attack correctly; or

3. participant did not recognise it was a combined attack.

We calculated the number of participants with each outcome, for each of the three attack combinations. These results are presented in Table 12, and show 
that of the 30 participants, 24 recognised that they had heard a combined attack, 16 of which identified both attacks correctly, and 8 only one of the attacks. The results show that almost all $(5 / 6)$ participants who did not recognise that it was a combined attack had been presented with the DDoS/port scan combination.

Table 12: Combined Attack Results

\begin{tabular}{|c|c|c|c|c|}
\hline $\begin{array}{l}\text { Attack } \\
\text { combination }\end{array}$ & $\begin{array}{l}\text { Number of } \\
\text { partici- } \\
\text { pants }\end{array}$ & $\begin{array}{l}\text { Outcome } \\
1\end{array}$ & $\begin{array}{l}\text { Outcome } \\
2\end{array}$ & $\begin{array}{l}\text { Outcome } \\
3\end{array}$ \\
\hline All combinations & 30 & 16 & 8 & 6 \\
\hline DDoS/port scan & 10 & 4 & 1 & 5 \\
\hline DDoS/data exfil & 10 & 6 & 4 & 0 \\
\hline Port scan/data exfil & 10 & 6 & 3 & 1 \\
\hline
\end{tabular}

\section{Hypothesis E: Intuitiveness of attack detection}

Fig. 8 and Fig. 9 are histograms of the click times in the pre- and posttraining tasks. The plots show that while detection was more accurate posttraining, the click times were clustered around the beginning of each attack in the pre-training task also. Tables 7 and 8 show that participants were mostly able to detect attacks in the pre-training task, with precision of 0.63 , recall of 0.96 and an F-score of 0.76 . Loss of connectivity had a significantly lower detection rate than the other attacks - we discuss the reasons for and implications of this in Section 7.

We assessed the pre-training attack-detection efficiency, by calculating mean detection times for each of the attack types in the pre-training task. The results are presented in Table 13. Compared with the post-training detection efficiency results presented in Table 9, the mean detection efficiency is similar (detection was slightly less efficient post- than pre-training for all attacks apart from DDoS). The standard deviation in detection efficiency is higher pre-training, indicating greater variance in the time taken to detect attacks before training.

Table 13: Pre-Training Efficiency of Attack Detection, in Seconds

\begin{tabular}{lll}
\hline Attack Type & $\begin{array}{l}\text { Detection time: } \\
\text { mean }\end{array}$ & $\begin{array}{l}\text { Detection time: } \\
\text { standard deviation }\end{array}$ \\
\hline DDoS & 2.53 & 2.32 \\
Port scan & 2.16 & 0.90 \\
Data exfiltration & 2.73 & 3.05 \\
Connectivity loss & 6.18 & 4.16
\end{tabular}

Participants were able to hear anomalies in the network traffic prior to training, but gave more false-positive responses at this stage than after training, as shown in Fig. 8. 
Hypothesis F: The effect of musical experience

We assessed the effect of musical experience on detection accuracy, detection efficiency, and identification accuracy, by comparing the results of participants who reported some level of musical training, to those without. Figs. 10 and 11 show the click times post-training of participants with musical experience, and without musical experience, respectively.

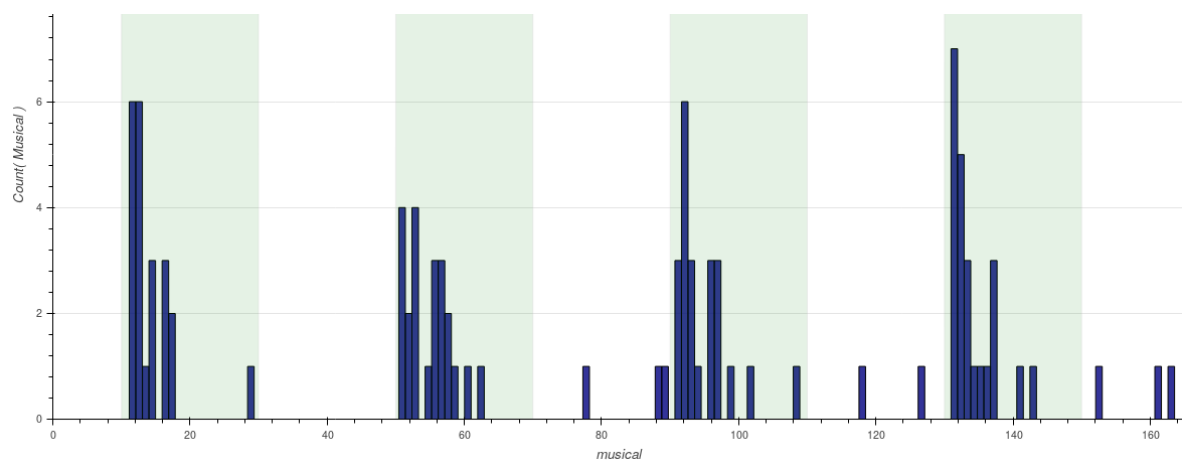

Figure 10: Participant Click Times in Post-Training Task: Participants With Musical Experience.

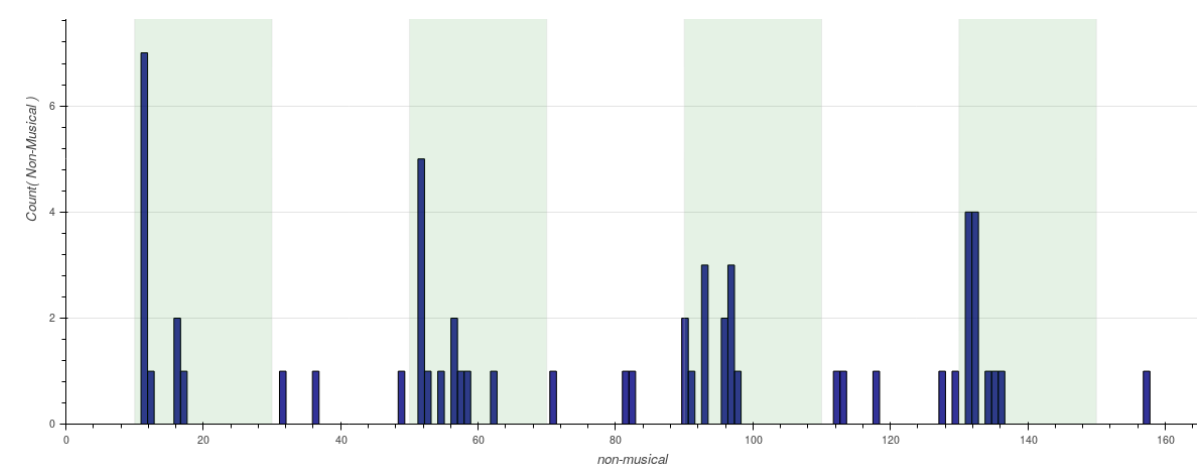

Figure 11: Participant Click Times in Post-Training Task: Participants Without Musical Experience.

\section{Detection accuracy}

The precision, recall and F-scores for participants with (20 participants) and without (10 participants) musical experience are compared in Table 14. The results show that while participants with musical experience detected attacks more accurately, the F-score for participants without musical experience is still reasonably high. 
Table 14: Accuracy of Attack Detection, Pre- and Post-Training Musical Experience Comparison

\begin{tabular}{lllllll}
\hline & \multicolumn{2}{l}{$\begin{array}{l}\text { Pre-training } \\
\text { Precision }\end{array}$} & Recall & F- & \multicolumn{2}{l}{ Post-training } \\
& & & Precision & Recall & F- \\
& & & & & & score \\
\hline Musical & 0.61 & 0.97 & 0.75 & 0.90 & 1 & 0.95 \\
Non-musical & 0.60 & 0.93 & 0.73 & 0.76 & 1 & 0.87
\end{tabular}

\section{Detection efficiency}

Descriptive statistics for the detection efficiency of participants with and without musical experience are presented in Table 15, and show that participants without musical experience had a slightly quicker mean detection time.

Table 15: Post Training Efficiency of Attack Detection: Musical Experience Comparison

\begin{tabular}{lllll}
\hline Variable & N & $\begin{array}{l}\text { Detection } \\
\text { time: mean }\end{array}$ & $\begin{array}{l}\text { Detection time: } \\
\text { dard deviation }\end{array}$ & stan- \\
\hline Musical & 20 & 3.68 & 2.32 & \\
Non-musical & 10 & 3.52 & 2.36 &
\end{tabular}

We ran a t-test to check for significant differences in the mean detection efficiency for participants with and without musical experience. We selected a two-sample t-test assuming equal variances. The variances were treated as equal based on the result on an initial F-test, which showed there was no significant difference in the variance in detection efficiency. The results of the t-test are displayed in Table 16. The results show that there was no significant difference in the mean detection efficiency between participants with and without musical experience with significance $\alpha=0.05$, since $t$ Stat $>-t$ Critical two-tail and $t$ Stat $<t$ Critical two-tail.

Table 16: t-Test: Two-Sample Assuming Equal Variances: Attack-Detection Efficiency for Participants With and Without Musical Experience (alpha=0.05)

\begin{tabular}{lll}
\hline & Non-musical & Musical \\
\hline Mean & 3.52 & 3.68 \\
Variance & 5.55 & 5.38 \\
Observations & 40 & 80 \\
Pooled Variance & 5.44 & \\
Hypothesized & 0 & \\
Mean Difference & \\
df & 118 & \\
t Stat & -0.36 & \\
$\mathrm{P}(\mathrm{T}<=$ t) one-tail & 0.36 & \\
$\mathrm{t} \mathrm{Critical} \mathrm{one-tail}$ & 1.66 & \\
$\mathrm{P}(\mathrm{T}<=\mathrm{t})$ two-tail & 0.72 & \\
$\mathrm{t}$ Critical two-tail & 1.98 &
\end{tabular}


Identification accuracy

Table 17 shows the identification accuracy results of participants with musical experience, and those of participants without musical experience. The results show that the incorrect port scan identification was made by 3 participants with musical experience, and 3 without.

Table 17: Post-Training Accuracy of Attack Identification: Musical Experience Comparison

\begin{tabular}{lll}
\hline Attack Type & $\begin{array}{l}\text { Participants with } \\
\text { musical experience }\end{array}$ & $\begin{array}{l}\text { Participants without } \\
\text { musical experience }\end{array}$ \\
\hline All attacks & $77 / 80$ & $37 / 40$ \\
DDoS & $20 / 20$ & $10 / 10$ \\
Port scan & $17 / 20$ & $7 / 10$ \\
Data exfiltration & $20 / 20$ & $10 / 10$ \\
Connectivity loss & $20 / 20$ & $10 / 10$
\end{tabular}

Musical experience had no significant effect on the ability of participants to use the sonification, either for detection or identification of attacks. The results show that participants with some level of experience playing a musical instrument performed slightly better than those without (see Table 14).

\section{Hypothesis G: Improvement through familiarity}

We compared the pre-training with the post-training accuracy and efficiency of detection. The results are shown in Tables 8 (accuracy) and 18 (efficiency). While there was a marked and continued improvement in detection accuracy throughout the four assessed datasets, there was little change in efficiency. Table 18 shows that detection time actually increased slightly throughout the datasets. Possibly, this is due to participants having more to think about, causing a delayed reaction time. Whereas in the initial task, participants were asked to click immediately if they heard anything abnormal, in the later tasks, in which they had knowledge of the attacks they should aim to detect and were asked to describe them verbally, it is likely that the process of deciding what the attack type was added to the detection time.

Table 18: Efficiency of Attack Detection: Dataset Comparison

\begin{tabular}{lll}
\hline Attack type & $\begin{array}{l}\text { Detection time: } \\
\text { mean }\end{array}$ & $\begin{array}{l}\text { Detection time: } \\
\text { standard deviation }\end{array}$ \\
\hline Pre-training & 3.18 & 3.11 \\
Training 2 & 3.19 & 2.30 \\
Training 3 & 3.46 & 2.21 \\
Post-training & 3.63 & 2.32
\end{tabular}




\section{Discussion}

\subsection{Study Results in the Context of SOCs}

We reflect on the results of the study, and consider the implications of these results for the use of sonification in SOC monitoring tasks. In general, the accuracy and efficiency of attack detection suggests there is value in further exploring the use of sonification in network-security monitoring tasks. The Fscore in the post-training task indicates that attacks were detected accurately using the sonification. We also showed that all attacks were detected in less than 3 seconds (except for loss of connectivity - the likely reason for the delay in detection of which we explained). This indicates that sonification of network traffic has potential as a method for enabling humans to detect attacks, and further research into a wider range of different attacks types and network sizes is worthwhile.

In real-world networks, attacks may occur simultaneously, either through coincidence, or because an attacker is deliberately causing a distraction from one attack using another. It was therefore important to test whether combinations of attacks could be heard, in order to assess the potential of the sonification tool for representing these conditions. Despite being given no indication during the study that a combined attack should be expected (see Section 5), 24 of the 30 participants recognised that they had heard a combined attack. Of the 6 who did not, 5 had been presented with the combined DDoS and port scan. This may have been due to the raised level of confusion between DDoS and port scan attack sounds across participants, which we describe in Section 7.3.

Participants detected attacks before training, by comparing sounds heard to a "normal" baseline sound. Despite not having heard the sounds of an attack before, participants were able to detect it using the sonification. The attack-detection approaches used in SOCs are often signature-based, and can only detect attacks for which signatures are already known. While statistical methods aim to avoid this reliance on prior knowledge of attacks, they can be inaccurate. The presentation of data such that humans are also involved in the detection process, and can recognise deviations from normal activity, even if those deviations have not been seen previously, is therefore beneficial. Based on the results of this study, sonification has potential as an approach to enabling this.

The minimal effect of musical experience on ability to use the sonification in this application is an important result, since to be suitable for use in realworld network-security applications, the system should be accessible to users regardless of their level of musical training. It is possible that attackers could deliberately launch attacks that "sound normal", in order to avoid detection by such methods. The proposed role of sonification is complementary to existing attack-detection tools, however, and further research into its use for widening the range of attacks that can be detected is important.

In summary, the results of the study indicate that sonification has the potential to be useful for network-attack detection, and could complement the existing attack-detection approaches of SOCs. Given that we based the network-traffic 
levels used on traffic levels from real network datasets, the approach is scalable to realistically-high network traffic levels. Assessment of the traffic-level limits of this approach will be important - particularly, the levels of network traffic that are required before attacks become masked by the sounds produced.

\subsection{Experience of Participants in the Study}

In an interview immediately following the study, participants were asked to rate the difficulty of each of the task breakdown components (detection of some attack; identification of musical change; identification of attack type) using a Likert scale, in which 1 was "very easy", and 5 "very difficult". Their responses are summarised in Table 19, and indicate that on average participants considered identification of the musical change and type of attack slightly more difficult than the initial detection of the attack. Some participants reported the types of attacks they had found it challenging to identify. The difficulty differentiating between port scan and DDoS was frequently noted at this stage, which supports our observations on the confusion between these two attacks.

Table 19: Participants' Ratings for the Ease of Each of the Study Task Breakdown Components (Likert Scale, 1: Very Easy - 5: Very Difficult

\begin{tabular}{llll}
\hline & $\begin{array}{l}\text { Detection } \\
\text { of some } \\
\text { attack }\end{array}$ & $\begin{array}{l}\text { Identification } \\
\text { of musical } \\
\text { change }\end{array}$ & $\begin{array}{l}\text { Identification } \\
\text { of attack } \\
\text { type }\end{array}$ \\
\hline Mean rating & 1.67 & 1.90 & 2.22 \\
Standard deviation & 0.70 & 0.77 & 0.95
\end{tabular}

All participants reported that the training session had helped them to use the sonification in the study. Some felt that the training had helped them only with the attack identification, while some reported that the training had also helped them with the initial identification of attacks. For example, "if there is no training, you can identify that something is wrong, but after a few minutes of training I could distinguish between traffic, outgoing, incoming traffic, so I can distinguish basically the source of the problem, if it's outgoing or incoming, and the type of attack quite easily I think". Asked about whether the training helped, for the initial detection, one participant stated: "it [the training] didn't help for detection that something was wrong, that was because it was obvious anyway", which suggests the participant felt able to hear anomalies in the network traffic equally well before and after training.

A number of participants stated that they felt the sonification was "intuitive" without training: "detection of an attack in sonification it's quite easy. Your brain captures the pattern of normal traffic quite fast, and you can distinguish the attack or not attack easily, even with no training". The ability of participants to hear anomalies without knowing what an anomaly "should sound like" (i.e., having as reference only the baseline sound of the network presented briefly at the beginning of the pre-training task) has important implications for the detection of network anomalies and attacks whose properties are unknown, that 
do not follow expected signatures or patterns, or which are zero-days. There is promise in exploring the use of sonification further for the detection, by listening humans, of attacks that do not match known profiles.

We observed during the study that some participants with a high level of musical experience appeared disadvantaged by a sensitivity to musical change that led to false-positive detections. However, some such participants also seemed able to learn the meanings of the sonification system such that they could report extra information over the attacks they had been trained to recognise, with one musically-trained participant reporting: "for abnormal stuff that you're not looking for, the most common that I'm hearing and that's throwing me off is higher range clarinet coming from this speaker, which would indicate to me then that something funny is going on in TCP with the outgoing destination ports". It is important to explore the effect of high levels of musical experience on ability to use sonification for network-security monitoring tasks.

\subsection{Sonification design}

The performance of participants in the study highlighted some design questions. A design aspect that caused some difficulty in the study was the sound of the piano representing packets sent over FTP. Some participants reported that the piano sounded abnormal, since the piano notes were too distinct. This caused a problem when occasional FTP transfers were present in the background

data, unrelated to the data exfiltration attack. Many of the false-positive clicks, during the pre-training task especially, correlated with the sound of piano notes that sounded abnormal to participants but in fact signalled small FTP transfers rather than attack traffic.

Participants frequently had difficulty distinguishing between the DDoS and port scan attacks: the only incorrect attack identification was the identification of the port scan as a DDoS (see Table 11). The most audible difference between the two was the difference in pitch range - DDoS being audible in the low pitch range, and port scan two octaves higher. The port scan had some sonic indicators of a DDoS: since it was an incoming attack from unusual IP addresses, there was low-range dissonance (beneath the high-range dissonant port sounds), which was also a DDoS indicator. Thus the sonification design may have contributed to the confusion.

When asked in the post-study interview whether there was anything they would change about the sonification design, the aspects noted most frequently by participants were the use of the pitch range mapping, and the background sound of the sonification. On the pitch ranges, one participant reported: "the thing that I found most difficult was deciding, is it high or low, the range", and the effect this had on participants' ability to differentiate between DDoS and port scan was also indicated: "for me between port scan and DDoS I had to always think which was it, was it port scan or is it DDoS". It was suggested by participants that a different mapping (not using pitch range) might be more appropriate for this data parameter, or a clearer difference between the low, medium and high pitch ranges by widening the range between them (greater 
than the octave between each used here). Assessing the effectiveness of these suggestions is left for further design experimentation

On the subject of the background noise of the sonification, one participant stated: "it was not my kind of music and I think that it was very annoying". However, some participants reported positively on the background noise "it was actually like music that I could listen to". This difference in opinion suggests the importance of addressing the sound design of the sonification by considering musical taste, and indicates that a choice of musical genres might be an appropriate approach.

\subsection{Study design}

There were some difficulties noticed in the study setup. Most obvious was the setup of the connectivity loss. In the sonification, a loss of connectivity was represented through silence, since as traffic ceased no sound events were generated. However, in the pre-training task especially, this caused some confusion for participants, some of whom assumed that the silence signalled the end of the task. Participants were instructed that the researcher would tell them when the dataset finished, and not to assume it had finished until that point, but there was still a level of confusion for some participants, and this seemed to affect the number of false-negatives for connectivity-loss detection (see Table 7).

When asked whether there was anything they would change about the study design, the aspects that participants noted most frequently were the difficulties in the loss of connectivity setup, and the positioning of the speakers. Participants suggested that the loss of connectivity setup would work well in practice, with the sudden silence being "striking", but that in the context of this study it was confusing: "I remain convinced that it would work very well in practice, so possibly some other indicator that experiment is going on, or something". Another participant suggested the use of a sonic indicator that the end of each dataset had been reached, such as a "beeping" sound. Some participants suggested that the positioning of the speakers used in the study should be different, or that they would have preferred to use headphones: "I think maybe the speaker placement should be a bit different. Because obviously that affects everyone's hearing differently, I mean I haven't got great hearing so I would probably try and get a bit closer".

There are potential limitations to the way in which true- and false- positive detections were measured (using the approach described in Table 3). In particular, since participants sometimes clicked multiple times during the same attack time windows, the measurement of what constituted a true-positive detection had to be considered. In calculating the efficiency of attack detection, we used the first true-positive click of the participant only. The calculation of precision and recall scores also helped to address this, since it showed the balance of true-positive clicks compared with false-positive, and is a measurement of participants clicking at any time during the attack window against outside the attack window.

In the datasets in which no verbal description of attack type was required by participants (the pre-training and training 2 datasets), it was not possible 
to know exactly what the participants believed they had heard. For example, it was unclear whether participants who clicked multiple times during the same attack believed that they had heard a new attack condition, or were clicking for the same one continuing. It was also not possible to know whether the participant was clicking the mouse, following the beginning of an attack in the dataset, because they had heard the indicators of that attack condition, or something else. For example, following a connectivity loss in the dataset, as the sound thinned, participants who clicked were recorded as having detected the connectivity loss, while it is possible that they in fact believed they had heard some anomalous sound other than the thinning of the sound.

It is difficult to avoid the above limitation, since it was essential to test the participant early on without describing the attack sounds, in order to assess the intuitiveness hypothesis, yet this naturally meant the participant was unable to describe the attack type. An approach might be to ask the participant in these early stages to describe the change in sound after each mouse click. However this may have placed extra strain on the participant having only just been introduced to the sonification. Our results suggest that in general participants were detecting the correct attack indicators in the early stages, since the Fscore, precision and recall across all attacks were good at this stage, indicating accuracy of detection.

Participants were asked to describe both the musical change and the attack type signalled. The aim here was to understand how they were completing the study tasks. We aimed to understand whether participants were memorising the sounds of the attacks (approach $A$ in Fig. 12), or were hearing the changes, contextualising their meaning in terms of the network data, and then deriving the type of attack (approach $B$ in Fig. 12). The distinction ties in with the intuitiveness hypothesis: for attacks that do not fit an expected profile, or are evolving in nature, it is important to assess whether participants are able to contextualise information and work out what is happening on the network using the sonification.

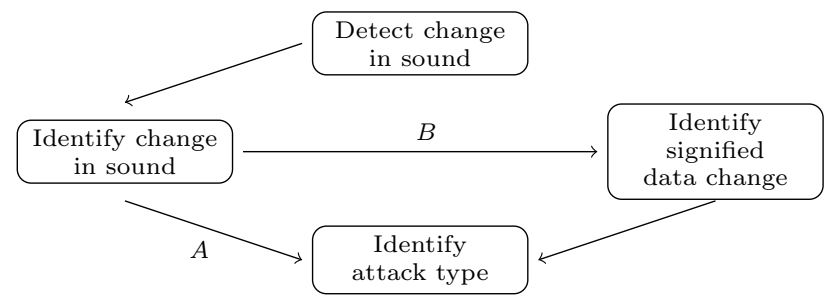

Figure 12: Possible Approaches to Completing the Study Task.

It appeared that there was a largely mixed approach: based on the researcher's observations in the study, some participants clearly memorised the attack types and sounds (approach $A$ ), and this is unsurprising given that only four attack types with distinctive sounds were used in this study. However, it also appeared that other participants were working from their knowledge of 
the data the sonification represented in order to work out the attack type, as in approach $B$. As discussed, participants were sometimes able to report extra information over what they had been taught in terms of attacks. This ability to derive further information suggests a depth to some participants' understanding of the sounds of the sonification and their meaning in terms of the network data.

In the post-study interview, some participants considered their method of completing the tasks. A number of participants reported that they had waited for some threshold amount of anomalous sound to play, before deciding whether an attack was happening. This was particularly for data exfiltration, since small amounts of outgoing FTP traffic were considered normal, while higher amounts indicated data exfiltration: "I think the hardest part was identifying, well once you pick the odd sound out, is identifying when that's like an actual persistent thing or not, or whether it's a one-off like the FTP thing still sounds out of place, but sometimes it might be ok".

Some participants also stated that they had used association of the sounds of the sonification with sounds from their background, to facilitate ease of recognition: "for me it's easier to think of them, again as I was saying jokily the 8-bit gameboy game, or the piano as opposed to trying to - I mean, go through more details of, the way you have put the different instruments in". The question of participants' task completion methods should be addressed more empirically in future, and participants could be asked to consider in more detail how they believe they are performing the detections.

\section{Conclusion and Future Work}

The results of this study support further research into the utility of sonification systems for network-security monitoring, by showing that it is viable for a set of attack conditions to be detected and identified accurately and efficiently using sonification. We showed that sonifying network data enables users to detect attacks, and to identify the types of attacks after training. We also showed that before training, abnormal network conditions could be heard intuitively to some extent, supporting the investigation of sonification for signalling unknown or evolving attack types, for which signatures do not exist. Combinations of attacks occurring simultaneously were audible in the sonification and recognised by a majority of participants. The performance of participants using the sonification was not significantly affected by their level of musical experience: participants without musical training were able to use the sonification.

We showed that there is benefit to using the sonification to "hear" attacks in network data without memorising the sound of the attacks (the "intuitiveness" hypothesis), but that memorising and using the sonification over time does improve performance. As future work, closer study of the effect of training and memorising sounds on performance using the sonification would enhance understanding of the way in which participants interact with sonification. Such

work should also assess whether the results we obtained - the intuitiveness of detection, and improvement over time - hold true in more complex systems and when representing more complex attacks. 
As future work, we intend to experiment with sonification designs for networksecurity monitoring, building on this prototype, the design questions highlighted by the study results (as discussed in Section 7), and the feedback of participants on their ability to understand the design. In particular, we will experiment with a range of real-world data sources, a variety of data-sound mappings, and different sonification aesthetics. As we discussed in Section 7.3, the pitch range sound parameter appears to have been especially difficult for participants to interpret. Future design experiments could identify more appropriate sound parameters for use in this context by comparing a range of possible sound parameters. Such sound parameters might include rhythm, timbre or articulation, for example, and audio phenomena such as the Shepard Tone might also be experimented with.

We aim to carry out studies to assess the use of sonification in realistic SOC tasks (in multitasking scenarios for example, to enable peripheral monitoring). We also intend to compare the attack-detection capabilities of analysts using sonification against their capabilities using other data-presentation tools, and to compare the performance of the sonification system against that of automated detection tools for a range of attack scenarios. We will also consider how this sonification technique performs in a real SOC environment, and intend to run the system in SOCs, to explore the performance of the approach alongside existing SOC tools and in the face of real-world scales of network data and attacks.

We intend to experiment with sonifying types of cyber attack other to those presented in this paper. This includes assessment of the effectiveness of sonification for signalling, for example, slower-moving malwares and advanced persistent threats (APTs). It would be valuable to experiment with a wider range of attack types and variations on attack types, to investigate the anomaly-detection potential of sonification for attacks for which indicators are not known. This research would explore the breadth of attack types that can be heard. It will also be important to explore the utility of sonification in helping users to distinguish between attacks and "rare" events that are not related to attacks. We anticipate that enabling users to link sonified data with a visual representation of that data may be important in enabling quick identification of whether the rare event was likely an attack or non attack, and intend to explore whether this is the case.

Another key area for future work, which fell outside the scope of this study, is testing the usability of sonification systems for network-attack detection. Such testing should assess whether the use of sonification systems in this context causes user fatigue, and also identify the preferred data-sound mappings of users. Prior work highlights the importance of usability testing for sonification approaches, and may guide approaches to such testing [49]. Furthermore, future work should explore the utility of such sonification systems to users with backgrounds outside cybersecurity and computer science. All participants in our study had knowledge in these fields; yet in order to understand the potential for the approach to be used by non-cybersecurity experts, studies in the future should include this demographic. 


\section{Acknowledgement}

The authors would like to thank the Engineering and Physical Sciences Research Council (EPSRC) for funding this work (grant number: EP/K035606/1).

\section{References}

[1] IBM X-Force Threat Intelligence Index 2017, https://www-03.ibm.com/ security/data-breach/threat-intelligence-index.html, 2017. [Online; accessed 15-June-2017].

[2] V. Chandola, A. Banerjee, V. Kumar, Anomaly detection: A survey, ACM Computing Surveys (CSUR) 41 (2009) 15.

[3] P. Garcia-Teodoro, J. Diaz-Verdejo, G. Maciá-Fernández, E. Vázquez, Anomaly-based network intrusion detection: Techniques, systems and challenges, Computers \& Security 28 (2009) 18-28.

[4] A. D'Amico, K. Whitley, D. Tesone, B. O'Brien, E. Roth, Achieving cyber defense situational awareness: A cognitive task analysis of information assurance analysts, in: Proceedings of the human factors and ergonomics society annual meeting, volume 49, SAGE Publications, pp. 229-233.

[5] A. DAmico, L. Buchanan, D. Kirkpatrick, P. Walczak, Cyber operator perspectives on security visualization, in: Advances in Human Factors in Cybersecurity, Springer, 2016, pp. 69-81.

[6] A. D'Amico, J. Goodall, D. Tesone, J. Kopylec, Visual discovery in computer network defense, IEEE Computer Graphics and Applications 27 (2007) 20-27.

[7] R. E. Etoty, R. F. Erbacher, A Survey of Visualization Tools Assessed for Anomaly-Based Intrusion Detection Analysis, Technical Report, DTIC Document, 2014.

[8] G. Kramer, B. Walker, T. Bonebright, P. Cook, J. H. Flowers, N. Miner, J. Neuhoff, Sonification report: Status of the field and research agenda. report prepared for the national science foundation by members of the internal community for auditory display (2010).

[9] T. Hermann, A. Hunt, J. Neuhoff, The sonification handbook, Logos Verlag Berlin, GE, 2011.

[10] L. Axon, J. Nurse, M. Goldsmith, S. Creese, A formalised approach to designing sonification systems for network-security monitoring, International Journal on Advances in Security 10 (2017).

[11] T. Hildebrandt, T. Hermann, S. Rinderle-Ma, Continuous sonification enhances adequacy of interactions in peripheral process monitoring, International Journal of Human-Computer Studies 95 (2016) 54-65. 
[12] M. Ballora, N. Giacobe, M. McNeese, D. Hall, Information data fusion and computer network defense, Situational Awareness in Computer Network Defense: Principles, Methods and Applications, Cyril Onwubiko, Thomas Owens, eds. IGI Global, Hershey PA (2012) 141-164.

[13] L. Axon, B. Alahmadi, J. R. C. Nurse, M. Goldsmith, S. Creese, Sonification in security operations centres: what do security practitioners think? (2018).

[14] M. Ballora, N. Giacobe, D. Hall, Songs of cyberspace: an update on sonifications of network traffic to support situational awareness, in: SPIE Defense, Security, and Sensing, International Society for Optics and Photonics, pp. 80640P-80640P.

[15] M. Gilfix, A. Couch, Peep (the network auralizer): Monitoring your network with sound., in: Proceedings of the Large Installation System Administration Conference, pp. 109-117.

[16] L. Qi, M. Martin, B. Kapralos, M. Green, M. García-Ruiz, Toward soundassisted intrusion detection systems, in: On the Move to Meaningful Internet Systems 2007: CoopIS, DOA, ODBASE, GADA, and IS, Springer, 2007, pp. 1634-1645.

[17] P. Vickers, C. Laing, T. Fairfax, Sonification of a network's self-organized criticality, arXiv preprint arXiv:1407.4705 (2014).

[18] M. Ahmed, A. N. Mahmood, J. Hu, A survey of network anomaly detection techniques, Journal of Network and Computer Applications 60 (2016) 1931.

[19] C. Tsai, Y. Hsu, C. Lin, W. Lin, Intrusion detection by machine learning: A review, Expert Systems with Applications 36 (2009) 11994-12000.

[20] J. Nicholls, D. Peters, A. Slawinski, T. Spoor, S. Vicol, J. Happa, M. Goldsmith, S. Creese, Netvis: a visualization tool enabling multiple perspectives of network traffic data (2013).

[21] T. Hinterberger, G. Baier, Poser: Parametric orchestral sonification of eeg in real-time for the self-regulation of brain states, IEEE Trans. Multimedia $12(2005) 70$.

[22] P. Janata, E. Childs, Marketbuzz: Sonification of real-time financial data, in: Proceedings of the International Conference on Auditory Display.

[23] D. Merced, L. Wanda, Sound for the exploration of space physics data, Ph.D. thesis, University of Glasgow, 2013.

[24] L. Axon, S. Creese, M. Goldsmith, J. Nurse, Reflecting on the use of sonification for network monitoring, in: Proceedings of the International Conference on Emerging Security Information, Systems and Technologies (IARIA), pp. 254-261. 
[25] A. Brown, M. Martin, B. Kapralos, M. Green, M. Garcia-Ruiz, Poster: Towards music-assisted intrusion detection (2009). Poster presented at IEEE Workshop on Statistical Signal Processing.

[26] R. Giot, Y. Courbe, Intention-interactive network sonification, in: Proceedings of the International Conference on Auditory Display, Georgia Institute of Technology, 2012, pp. 235-236.

[27] V. F. Mancuso, E. T. Greenlee, G. Funke, A. Dukes, L. Menke, R. Brown, B. Miller, Augmenting cyber defender performance and workload through sonified displays, Procedia Manufacturing 3 (2015) 5214-5221.

[28] C. Papadopoulos, C. Kyriakakis, A. Sawchuk, X. He, Cyberseer: 3d audiovisual immersion for network security and management, in: Proceedings of the ACM workshop on Visualization and data mining for computer security, ACM, pp. 90-98.

[29] P. Varner, J. Knight, Monitoring and visualization of emergent behavior in large scale intrusion tolerant distributed systems (Technical report, Pennsylvania State University, 2002).

[30] M. Debashi, P. Vickers, Sonification of network traffic flow for monitoring and situational awareness, PloS one 13 (2018) e0195948.

[31] M. Debashi, P. Vickers, Sonification of network traffic for detecting and learning about botnet behaviour, IEEE Access (2018).

[32] M. Gopinath, Auralization of intrusion detection system using Jlisten, Development 22 (2004) 3.

[33] A. de Campo, A data sonification design space map, in: Proc. of the 2nd International Workshop on Interactive Sonification, York, UK.

[34] S. Barrass, Sonification design patterns, in: Proceedings of the International Conference on Auditory Display, Georgia Institute of Technology.

[35] S. Bly, Presenting information in sound, in: Proceedings of the 1982 conference on Human factors in computing systems, ACM, pp. 371-375.

[36] T. Hermann, Sonification for Exploratory Data Analysis, Ph.D. thesis, 2002. Bielefeld University.

[37] MAWI working group traffic archive, http://mawi.wide.ad.jp/mawi/, 2017. [Online; accessed 15-March-2017].

[38] Trace statistics for CAIDA passive OC48 and OC192 traces, http:// www.caida.org/data/passive/trace_stats/, 2016. [Online; accessed 15March-2017].

[39] M. Bhuyan, D. Bhattacharyya, J. Kalita, Surveying port scans and their detection methodologies, The Computer Journal (2011) bxr035. 
[40] K. Vishwanath, A. Vahdat, Swing: Realistic and responsive network traffic generation, IEEE/ACM Transactions on Networking (TON) 17 (2009) 712725 .

[41] D. Lee, B. Carpenter, N. Brownlee, Observations of udp to tcp ratio and port numbers, in: Internet Monitoring and Protection (ICIMP), 2010 Fifth International Conference on, IEEE, pp. 99-104.

[42] B. Chandrasekaran, Survey of network traffic models, Waschington University in St. Louis CSE 567 (2009).

[43] Center for applied internet data analysis (CAIDA), http://www.caida. org, ???? [Online; accessed 24/02/2018].

[44] P. Barford, D. Plonka, Characteristics of network traffic flow anomalies, in: Proceedings of the 1st ACM SIGCOMM Workshop on Internet Measurement, ACM, pp. 69-73.

[45] SuperCollider, supercollider.github.io, 2017. [Online; accessed 30January-2019].

[46] C. Goutte, E. Gaussier, A probabilistic interpretation of precision, recall and f-score, with implication for evaluation, in: European Conference on Information Retrieval, Springer, pp. 345-359.

[47] G. Creech, J. Hu, Generation of a new ids test dataset: Time to retire the kdd collection, in: Wireless Communications and Networking Conference (WCNC), 2013 IEEE, IEEE, pp. 4487-4492.

[48] A. Field, Discovering statistics using IBM SPSS statistics, Sage, 2013.

[49] A. Siami Namin, R. Hewett, K. S. Jones, R. Pogrund, Sonifying internet security threats, in: Proceedings of the 2016 CHI Conference Extended Abstracts on Human Factors in Computing Systems, ACM, pp. 2306-2313. 